\title{
ELECTRICAL BEHAVIOUR OF LANGMUIR FILMS: A REVIEW, PART I
}

\author{
V.K. AGARWAL $\dagger$ \\ International Centre for Theoretical Physics, Trieste, Italy.
}

(Received July 1, 1974; in final form August 22, 1974)

The electrical behaviour of thin films obtained by a variety of processes, e.g. thermal evaporation in vacuum, have been extensively studied. However, the study of organic mono- and multilayer films obtained by the Blodgett-Langmuir technique (commonly referred to as Langmuir films) has gained considerable momentum only during the past decades. Unlike evaporated films, the striking features of these organic films are their controllable thicknesses down to one monolayer $(\sim 25 \AA)$ and the possibility of obtaining them free from holes and conducting imperfections. The aim of this paper is to describe the film deposition techniques, some of the properties of the films so obtained and to review their electrical behaviour. It is also intended to make this review a comprehensive and up-to-date source of information for those who are either already engaged in this field or are planning to make Langmuir films the subject for future investigations.

In this survey, emphasis is put on problems worth further study to obtain greater insight into the basic properties of these films. Further, since they possess some interesting electrical properties, this paper may prove useful in the assessment of our depth of knowledge about them and in reducing the existing gap between basic research and technological applications. Thus their potential usefulness in the development of devices is also discussed.

The survey is divided into two parts. Part $I$ is concerned with deposition techniques, the physical properties of Langmuir films and certain electrical properties, namely dielectric behaviour and electrical conduction phenomena. Part II, which will appear in the next issue, will be concerned with electrical breakdown behaviour, voltage induced changes in electrical behaviour (forming) and ionic transport phenomena in the films and will finish with suggestions as to future trends in work with such films together with a summary of possible applications.

To assist the reader, the two parts have been numbered consecutively with regard to sections of the text, figures and references.

1. Introduction

2. Film Deposition Techniques
2.1 Blodgett-Langmuir Technique
2.1.1 List of precautions
2.1.2 Selection of substrate and cleaning procedure

2.2 Evaporation method

3. Physical Properties of Langmuir Films

3.1 Nature and Orientation

3.2 Uniformity and Thickness.

3.3 Film Structure

3.4 Defect Nature

3.5 Skeletonization Process

4. Dielectric Properties
4.1 Early Measurements
4.2 Recent Measurements
4.3 Theoretical Work
4.3.1 Monomolecular films
4.3.2 Multimolecular films
4.4 Dielectric Loss Measurements

$\dagger$ Present address: Research Institute of Electronics, Shizuoka University, Hamamatsu-432, Japan. 
5. Electrical Conduction Properties

5.1 DC Conduction Theories in Thin Films

5.1.1 Fowler-Nordheim type tunnelling

5.1.2 Richardson-Schottky effect.

5.1.3 Space charge limited currents .

5.1.4 Poole-Frenkel effect

5.2 Conduction Mechanisms in Langmuir Films

5.3 High Field Conduction Characteristics

References .

KEY:

$\epsilon=$ dielectric constant

$C=$ capacitance

$d=$ monolayer thickness

$N=$ no. of monolayers transferred

$A=$ area of the film capacitor

$n=$ number of carbon atoms in the molecular chain

$I=$ current

$V=$ voltage

$k=$ Boltzmann's constant

$T=$ absolute temperature

$\psi=$ work function

$f=$ frequency

$J=$ current density

$e=$ electronic charge

$\mathrm{n}=$ Planck's constant

$m=$ electronic mass

$\mu=$ electron mobility

$\phi=$ potential barrier height

$\chi_{i}=$ work function of the insulator

$x_{m}=$ work function of the metal

\section{INTRODUCTION}

Insulating thin films in the thickness range $100 \AA$ to $20,000 \AA$ have been of interest to the scientific community because of their potential applied significance for developing optical, magnetic and electronic devices. Some of the unusual electrical properties possessed by thin films, which are unlike those of bulk materials, led to ideas about their technological applications and consequently interest in thin film studies grew rapidly. Earlier studies were not very inspiring because the films obtained always had pinholes, stacking faults and other impurities, and hence the results were not reproducible. In the last few decades, however, many sophisticated methods have become available for the production and examination of thin films, and greater reproducibility can be achieved. Nevertheless, the unknown nature of inherent defects in a wide variety of thin film systems still complicates the interpretation of many experimental data and thus limits their use in devices.

In a recent bibliographical survey on breakdown conduction in thin films, ${ }^{1}$ the author found that the major subject of investigation have been films pre- pared by thermal evaporation in vacuum. Langmuir films have remained less known among workers in this field. In fact, films formed by oil on water surfaces were known as early as 1774 when Benjamin Franklin reported their presence. Lord Rayleigh ${ }^{2}$ was the first to propose that these films were only one molecule thick. Rayleigh's hypothesis of monomolecular spreading thus gave birth to a new branch of science involving the study of the physics and chemistry of surfaces ${ }^{3}$. Subsequently, Langmuir ${ }^{4}$ introduced the simple technique of transferring these monolayers onto a substrate by moving the latter across the film-covered surface. This technique of transferring the monolayers onto the solid surface by a repeated dipping and withdrawal process was later modified by Blodgett and Langmuir ${ }^{5,6}$ and has become the basis of a new field of thin film investigations. This novel technique of "building-up" mono- and multilayer films is commonly known as the Blodgett-Langmuir (henceforth B-L) technique, and the films thus formed are often referred to as Langmuir films. $\dagger$

Using this simple technique, it is now possible to obtain monomolecular spreading of a number of organic substances, such as fatty acids and their soaps, branched and unsaturated fatty acids, esters, dyes and many proteins.

It appears that this is the only technique by which it is possible to deposit films on smooth substrates which are uniform, structurally oriented and whose thicknesses are accurately known and controllable. It is now well established that films which are largely free from defects and other conducting imperfections can be obtained, and a wide range of electrical characteristics can be studied in a variety of organic materials in thin film form. However, we shall here be concerned only with "solid" monolayers in which the molecules are closely packed, and which can be deposited on solid surfaces. Among the many substances which form a stable monolayer, the straight

†These films are also quoted as 'L. B. films' and the technique as the Langmuir-Blodgett (L. B.) technique' in the literature elsewhere. 
chain fatty acids and their metal soaps are used for most electrical measurements. This is probably because the fatty acids precipitated by calcium or barium ions in the solvent were used initially ${ }^{4,5,6}$ and the technique was perfected for them.

Although in principle any substance forming an insoluble, stable and condensed monolayer can be used for building up multilayers, in practice this is not necessarily so. For example, the molecules containing a hydrophilic group such as $-\mathrm{OH}$ or $-\mathrm{COOH}$ at one end of the chain and a hydrophobic group such as $\mathrm{CH}_{3}$ at the other have been found to form good monolayers, the molecules being arranged almost vertically on the water surface and becoming closely packed when the monolayer is compressed. But not all such substances behave this way. If the molecule is too short, the Van der Waals forces along their lengths will be insufficient to hold them together as a "solid" film and with long molecules, the buckling of the molecules themselves may give rise to a crumpled film on compression. Therefore, only molecules of intermediate chain length can be used to obtain an oriented, close-packed, solid monolayer on the water surface. However, there are no clear cut criteria for the choice of substances forming good Langmuir films. Reference should be made to the excellent text of Gains ${ }^{7}$ for the choice of material, and a recent review by Srivastava ${ }^{8}$ is a comprehensive source of information for beginners in this field. In addition, the reader is referred to several more reviews and texts 9-14 that have appeared.

Langmuir films were successfully prepared as early as 1935 but study of their dielectric properties at that time did not yield reproducible results. Renewed at tempts were made in the sixties but it was not until late sixties that reliable results were obtained. This was because many further advances in the original B-L technique were made during the last decade to obtain a good film for reliable electrical measurements. A handful of experimental data on electrical behaviour of Langmuir films during the last few years has yielded some very interesting electrical characteristics which will be discussed exhaustively in this paper. There is no other useful survey available in the literature giving an up-to-date account of their electrical behaviour. Even books and reviews published recently or in the past covering the whole subject of thin films do not mention work on Langmuir films. The one review devoted to Langmuir films ${ }^{8}$ contains only limited information about their electrical characteristics.

Essentially, the two crucial factors governing thin film properties are their uniformity of thickness and structural perfection. Particularly, all the electrical properties are very sensitive to these parameters and thus, for a judicious experimental study, one requires films of accurately known thickness and free from gross defects. Langmuir films are known to fulfill these requirements to a far greater extent than other types of thin films. For instance, Mann and Kuhn ${ }^{15}$ have recently shown through their electrical studies that the films obtained by the modified B-L technique were free from any holes and conducting imperfections. Secondly, the uniform thicknesses of these films are accurately known, as measured by standard optical methods like Tolansky's multiple beam interferometry, ${ }^{16,17}$ and may be controlled and reproduced successfully to as little as one molecule thick (about $25 \AA$ ). In addition to many other features described in the text of the paper, these two constitute a sound basis for studying these films. Control of these two parameters in other film systems is notoriously difficult to achieve.

To fulfill the aim of this paper, the film deposition technique of Blodgett and Langmuir ${ }^{5,6}$ will be discussed in detail. The recently proposed evaporation technique ${ }^{18}$ to obtain such organic films down to one molecule thick and with better purity than the evaporant, will also be described. The physical properties of these films, which are relevant in the context of their electrical behaviour, are described in the next section. Each of the following sections cover a particular aspect of the electrical behaviour and includes a detailed account of experimental and theoretical work. To make this survey more substantial, it has been thought desirable to discuss briefly the existing theories about conduction mechanisms, breakdown behaviour, etc., in the appropriate sections. This will certainly prove useful for the better understanding of the subject and may further help the researchers to select suitable problems for their studies on Langmuir films.

As many reviews and books have already been published on the whole range of electrical measurements in thin films, this paper is devoted to Langmuir films only. However, to adopt a broad viewpoint, some pertinent references have been cited to make comparisons between the results on Langmuir films and on other film systems. To make the survey an up-to-date source of information, all possible references since 1937 (when the earliest work on electrical properties was reported) have been provided.

Finally, some space has been reserved to discuss briefly the future potential of these films in the development of devices. In spite of the fact that further electrical studies on Langmuir films would 
prove useful for a better understanding of the underlying electrical phenomenon in thin films, it is also very likely that the use of such ordered film systems in developing devices could lead to a major breakthrough in the advancement of thin film technology.

\section{FILM DEPOSITION TECHNIQUES}

\subsection{Blodgett-Langmuir Technique}

This technique requires a very simple apparatus consisting of a long narrow trough $\mathrm{T}$ (Figure 1) usually

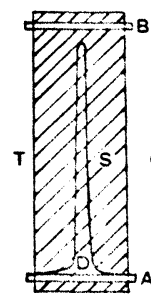

(a)

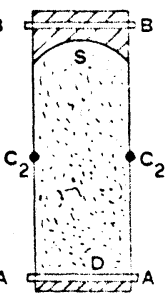

(b)

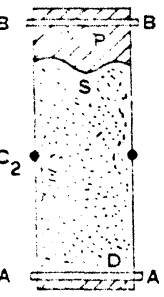

(c)

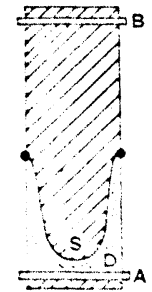

(d)
FIGURE 1 Diagrammatic representation of the apparatus for building up the Langmuir films [modified after Langmuir ${ }^{20}$ ?.

made of inert materials such as teflon, perspex, etc. Metals such as copper or aluminium are not used because they may contaminate the solution with metallic ions whose presence would stop the deposition. The inside of the trough is heavily waxed $\dagger$ this waxing is necessary in order to obtain a higher water level at the edges, which ensures that there is no leakage of the monolayer past the barriers. The barriers, rectangular in shape, such as glass slides, must also be waxed all around to render them hydrophobic. The trough is first levelled and then filled to the brim with doubly distilled or deionized water (specific resistance $\simeq 6 \times 10^{5} \Omega \mathrm{cm}$ ), having a low concentration of barium by adding $3 \times 10^{-5} \mathrm{M}$ barium chloride or the like. For easier deposition, the $\mathrm{pH}$ of the solution is adjusted to greater than 6 by the addition of a specific quantity of an alkaline substance. For example, $\mathrm{KHCO}_{3}$ at a concentration of $4 \times 10^{-4} \mathrm{M}$ holds the $\mathrm{pH}$ at 7.2. This results in smooth deposition of Y-type films. The surface of the liquid is now thoroughly cleaned by sweeping the waxed barrier $B$ across the long edges of the trough and finally leaving it resting on the trough end (Figure 1a). Another barrier, A, rests at the other end of the trough which is used to hold the waxed silk

HIn the case of teflon trough, the waxing is not required. thread $S$ placed upon the water surface as shown between A and B. The thread is waxed so that it does not wet and sink into the water, and is carefully made to touch the water surface at every point to avoid any possibility of leakage of the spread monolayer. The technique here is specifically for barium stearate films, but is similar for all other potential Langmuir films.

Stearic acid (or other material to be deposited) dissolved in benzene (concentration $\simeq 3 \times 10^{-4}$ by weight) is placed by the micrometer syringe on the water surface near D in the form of a small droplet which spreads spontaneously and pushes the thread outwards to take the shape as shown in Figure 1b. (If the first drop does not spread quickly, it is an indication that the surface is not perfectly clean.) As soon as the spreading is complete, the thread is fastened to the trough edges by two small clips $C_{2}$. At this stage the divalent barium ions present in the solution undergo surface reaction with stearic acid molecules to form the barium stearate soap, and the solvent (benzene) slowly evaporates, leaving behind a monolayer on the surface, its boundary being confined by the thread. If a second drop is put near D after the spreading is complete, it would not spread but remain as a residue, which is an indication that the available surface has been covered with the monolayer. The stearate molecules at this stage remain standing upright with their reactive carboxyl groups touching the water surface, but some of them also tend to bend over the water surface due to the relatively large space available for them. A small drop of oleic acid called "piston oil" is now placed on the surface at $\mathrm{P}$ which thus exerts a constant lateral compression of $30 \mathrm{dyn} / \mathrm{cm}$ on the spread monolayer. It has been found that this value of surface pressure $(\sim 30 \mathrm{dyn} / \mathrm{cm})$ is sufficient to compress a mono-layer of stearic acid into a solid phase with the molecules relatively closely packed. The thread boundary now separates the whole surface area between barriers $\mathrm{A}$ and $\mathrm{B}$ into two regions, with a stearic acid monolayer on one side of the thread and oleic acid on the other, these pressing each other to give an equilibrium shape to the thread (shown in Figure 1c). The transfer of the monolayer onto the "conditioned" slide may now be accomplished by moving the dipper near D up and down repeatedly across the monolayer covered surface. "Conditioning" here means that the slide has been made hydrophobic. This is achieved simply by rubbing the slide surface with a waxy solid, e.g. ferric stearate. It has been found that a pure aluminium or silver film deposited on the cleaned slide by thermal evaporation 
also behaves as a hydrophobic surface for good multilayer deposition.

The monolayer is thus transferred onto the "conditioned" slide on both its downward and upward journey, and the process may be continued until the thread S moves forward through an area to attain the shape shown in Figure 1d. Every time the thread moves forward through an area equal to that of the slide on which the film is deposited, the thread motion takes place because of the constant compression provided by the "piston oil." This visible motion of the thread is a very clear cut indication that the layers are being transferred on to the slide every time it is dipped or withdrawn. The film thus built up is termed "Y-type", and contains an even number of layers. Film thickness can then be accurately calculated by counting repeatedly the number of monolayers, and multiplying it by the known monolayer thickness. To obtain an odd number of monolayers outside the water surface, it is necessary first to dip the slide in the solution and then to spread the stearic acid - as shown in Figure 1b. In this case, the first layer will be transferred when the slide is moved upwards, and finally an odd number of layers will be obtained. Even to obtain one monolayer, this process has been found satisfactory by the author in his studies of these films. To obtain X type films, it is necessary to make the solution more strongly alkaline $(\mathrm{pH}=9)$ and then the transfer will occur only during the downward movements of the slide. It is necessary to maintain the ambient temperature at $20-22^{\circ} \mathrm{C}$ during experimentation, and the substrate should be raised or dipped across the monolayer very slowly and smoothly. To obtain a large number of layers on a solid substrate, i.e. thicker films, the author has found that use of a trough of large dimensions, and thus a large surface area for monolayer spreading, is not effective because the perfect cleaning of the surface becomes more difficult, and the molecules develop a tendency to collapse. More satisfactory results have been obtained by using a trough of smaller dimensions (say $29 \times 20 \times 6 \mathrm{~cm}$, as used by Nathoo ${ }^{19}$ ) and using a fresh solution after every deposition cycle is completed. It is also advisable to prepare the solution all at once in a large quantity to avoid any possible changes in its $\mathrm{pH}$ value, which may affect the nature of monolayer deposition.

The technique described above appears attractively simple in principle. However, it requires experimental skill to obtain structurally stable and smooth films. It is therefore necessary to mention some of the important precautions to be taken during film pre- paration. For further details reference should further be made to the text book of Gains ${ }^{7}$ and to the original papers of Blodgett and Langmuir ${ }^{5,6,20}$ One can also make use of commercially available highly sophisticated film balances ${ }^{13}$ for film deposition.

\subsubsection{List of Precautions}

1) The wax and chemicals used in the experimentation must be of highest quality and of extreme purity. For still higher purity, the solvents (benzene, etc.) should be fractionally distilled.

2) The water used should have good conductivity. For best results deionized water should be treated with $\mathrm{KMnO}_{4}\left(10^{-3} \mathrm{M}\right)$ and $\mathrm{NaOH}\left(10^{-2} \mathrm{M}\right)$ and after heating it for about 10 hours at $70^{\circ} \mathrm{C}$, it must be doubly distilled in a quartz apparatus. It must be remarked here that deionized water, if used directly, may contain some organic impurities.

3) Greatest care must be taken against dust contamination as the introduction of any foreign material from the outside may cause disruption of the film structure. It is thus necessary to cover the whole experimental set-up with a suitable transparent shield box and to design it in a way that all the operations needed during experimentation, e.g. the movement of the dipper, be operative from outside.

4) Any sort of mechanical vibrations or other disturbances during deposition may result in cracks in the deposited film giving rise to voids and inhomogeneities in the film. For this, the experiment should preferably be performed on a rigid foundation or, if a table is used, anti vibration mountings must be used.

5) The required $\mathrm{pH}$ value must be maintained accurately by adding the specified concentrations of the substances. Any change in its $\mathrm{pH}$ may markedly affect the nature of the deposited film. Specifically, the barium ion concentration should be properly maintained to avoid the presence of free stearic acid molecules in the deposited film.

6) Since the hands of the workers are themselves the most likely source of greasy contamination, it is necessary to clean them as thoroughly as possible. Specific attention is also required to avoid any surface active contamination.

7) Film transfer should be accomplished under as high a lateral compression as possible. The higher the surface pressure, the more compact will be the film. The use of oleic acid as "piston oil" ensures a satisfactory film deposition without voids.

8) The first monolayer should be transferred as slowly as possible to obtain a smooth surface, because 
it acts as the foundation for building-up the multilayers. It is also essential that the slide be completely dry before each immersion.

Since the whole phenomenon is on a molecular level, even small concentrations of impurities or contamination in any form may affect the results markedly. Therefore, the key-words in the preparation of Langmuir films are the purity and cleanliness of all the components used. For greater details one should refer to the recent work of Nathoo ${ }^{19}$ who has described at length the preparation of Langmuir films for electrical studies. One novel feature which has been introduced by Nathoo ${ }^{19}$ is the use of a device to raise and lower the substrate across the monolayer-covered surface. The PTFE piston used for the device is pulled upwards by the suction of a pump and falls under gravity when the suction is reduced. This device can control the speed of the dipper in the range $0.1 \mathrm{~mm} / \mathrm{s}$ to $1 \mathrm{~mm} / \mathrm{s}$.

\subsubsection{Selection of substrate and cleaning pro-} cedure Obviously, one of the major factors governing the degree of uniformity of the deposited film in the above process is the smoothness of the substrate used. Therefore, considerable attention must be paid to the selection of a substrate with smooth surfaces, and subsequent thorough cleaning to minimize the appreciable variations in the electrical properties which can occur. Microglass slides having no scratches are first selected and rinsed thoroughly with deionized distilled water. The planeness and smoothness of the selected slides are then checked by the standard method of matching their surfaces with a master optical flat ${ }^{21}$. On proper illumination, the formation of reasonably straight, equidistant, parallel and smooth fringes show that the slide surfaces do not have much curvature and are almost plane $\mathrm{e}^{22}$. The smoothness of such selected microglass slides can be of a much higher degree than that of the usual optical flat ${ }^{22}$. This is, for example, revealed by the absence of "wriggle" in the fringes of equal chromatic order (FECO) in extensive film thickness measurements on a 4-layer barium stearate film" ${ }^{16}$ using "Gold Seal" microslides. The author himself has used "Gold Seal" microslides in electrical measurements and found them satisfactory for obtaining uniform Langmuir films.

The selected microglass slides are then treated with chromic sulphuric acid (cold saturated), rinsed with doubly distilled water and immersed in caustic soda solution for several hours. Finally, they are thoroughly washed using a strong jet of doubly distilled water and dried by a current of warm air.
Ultrasonic cleaning with Ultrax cleaning fluid, and washing with distilled water also gives satisfactory cleaning. Both of these methods are used by Bücher et $a l^{23}$ in their work in building-up monolayers of arachidic acid. The degree of cleanliness of a slide may then be checked by immersing it through a cleaned water surface covered with a small amount of talcum powder, lycopodium or such like. The presence of any greasy contamination on the slide will be detected by the grains of talcum being pushed away when the slide is immersed. This simple test was devised by Langmuir ${ }^{24}$ and may also be applied to control the cleanliness of all glass components used for the experiment. Absence of any "breath figures" on the slide after breathing on it is also a good test of the cleanliness.

\subsection{Evaporation Method}

The recent attempt by Baker ${ }^{18}$ to deposit thin films (of the order of one monolayer thick) of organic solids by thermal evaporation in vacuum opens up a new field. It was reported by Luff and White ${ }^{25}$ that thermal degradation of high molecular weight materials takes place during vacuum evaporation, whereas thermal effects are much lower in the case of lower molecular weight solids. Thus it was realized that thermal decomposition of stable compounds is less likely to occur. Making use of these arguments, Baker was successful in depositing thin films of stearic acid (mol. wt $\cong 284.5$ ) and melissic acid (mol. wt $\cong 466.8$ ) by thermal evaporation in vacuum. The unchanged composition of both the evaporated films and the evaporant residue are evidence for the absence of thermal degradation in this case. Baker obtained very thin films of the order of one monolayer in thickness by maintaining a slow controlled rate of evaporation and other experimental requirements. It has also been pointed out by him that the purity of the remaining evaporant and evaporated films was slightly higher than the acid salts originally used, and has ascribed it to be due to the "outgassing" of the acid prior to deposition. Whereas this technique is of particular interest in the field of tribology because of the possibility of producing a monolayer film of boundary lubricant, the author feels that it is of equal importance for the physicists to study the electrical characteristics of such evaporated films. Although Baker has not mentioned explicitly the defect nature of these evaporated organic films, the absence of thermal decompositon and reported better purity are the two important features. 


\section{PHYSICAL PROPERTIES OF LANGMUIR FILMS}

Many interesting physical properties of Langmuir films have been investigated and these have been reviewed well in the existing literature ${ }^{8}$. In this section, only those properties which have relevance to the electrical behaviour are discussed briefly.

\subsection{Nature and Orientation}

The nature and orientation of the deposited film is mainly governed by the angle of contact between the monolayer-covered solution and the solid surface; hence the condensed monlayers may be obtained, at will, with different molecular orientation. These monolayers are characterized as $\mathrm{X}, \mathrm{Y}$ or $\mathrm{Z}$ types, and their molecular arrangements are shown in Figure 2.

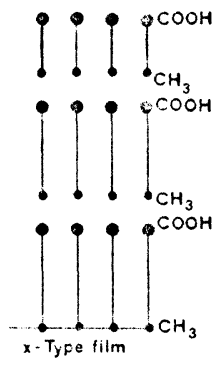

(a)

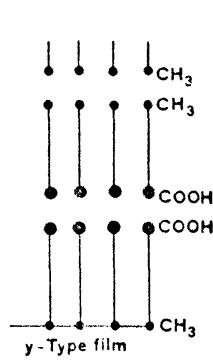

(b)

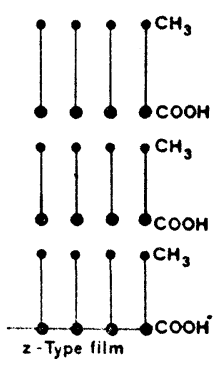

(c)
FIGURE 2 The molecular orientation of built-up Langmuir films. (a) $\mathrm{X}$ type (b) $\mathrm{Y}$ type; and (c) $\mathrm{Z}$ type.

The molecules in the case of $\mathrm{X}$ and $\mathrm{Z}$ type films are oriented in the same direction and thus the surface of the film will be composed of carboxyl and methyl groups, respectively. On the other hand, in a Y type film, the molecules in adjacent layers are oriented in opposite directions, and the film surface is composed of methyl groups. Of these three types of films, the one which has been studied is the $\mathrm{Y}$ type in which the monolayer transfer takes place both ways, i.e. on each dipping and withdrawal of the slide across the surface. On the other hand, in X films, transfer takes place only when the slide is being dipped and for $\mathrm{Z}$ type, it takes place only when the slide is withdrawn. The orientation is termed "exotropic" when the methyl groups touch the solid surface and the carboxyl groups remain away from it in the first monolayer. If the orientation of these two groups is reversed, the monolayer is termed "endotropic".
Thus, an $X$ type film is made up of a series of exotropic layers; the $\mathrm{Y}$ type is made up of a series of alternating exotropic and endotropic layers, and the $\mathrm{Z}$ type is made up of a series of endotropic layers. However, it must be pointed out that $Z$ type films are rather uncommon.

\subsection{Uniformity and Thickness}

Two crucial factors for making satisfactory electrical measurements on films are their uniformity and accurate knowledge of their thickness. Both of these requirements are fulfilled to a greater extent if Langmuir films are used. The thickness of several fatty acid monolayers has been measured using the best known optical methods. For example, a significant contribution in this context has been due to Srivastava and Verma ${ }^{16,26}$. They ${ }^{16}$ have employed Tolansky's standard multiple beam interferometric technique with fringes of equal chromatic order (FECO), which ensured absence of any error in their measurements due to surface irregularities. They thus obtained extremely sharp and smooth fringes, and determined thicknesses with higher accuracy. Subsequently, "C" spacings of these films were also measured by the same workers ${ }^{26}$ using the microfocus X-ray technique and excellent agreement was found between $\mathrm{C}$ spacing values and those obtained by interferometry. Recently, Scott and Sirhata ${ }^{17}$ have used the above technique with some modifications. They employed a protective collodion layer in measuring thicknesses, which improved the quality of the fringes and hence the precision of the measurements. Use of the protective layer thus avoided damage of the monolayer in vacuum, which is expecied to have a marked influence on the overcoating deposited. In fact, it was indicated 27,28 that over $60 \%$ of stearic acid film is removed by exposure to vacuum for 30 minutes.

As far as the uniformity of these films is concerned, it has already been stressed that the selection of the substrate must be made carefully. However, it has been shown by Holt ${ }^{29}$ that increasing the number of monolayers increases the degree of uniformity of the film. To achieve the required uniformity the deposition of the first monolayer is very important, since any voids or imperfections in it may lead to major disruption of the subsequent layers. Therefore, one must make sure that the slide emerging from the solution is completely dry before being re-immersed.

Whereas films of barium copper stearate could be built up containing as many as 3000 layers simply by repeated dipping and withdrawal processes 5,6 , some 
problems were encountered when thick multilayers of other fatty acid salts were deposited. Blodgett and Langmu ir ${ }^{6}$ noticed a "fogged" appearance for thicker barium stearate films (300 layers) and also observed the "cracking" tendency of these films, which increased with thickness. They then indicated that the addition of copper ions $\left(\cong 2 \times 10^{-6} \mathrm{M}\right)$ to the solution avoided both these difficulties and facilitated film deposition. Another factor which crops up during deposition is the gradual crumpling or collapse of the stearate monolayers. Henke ${ }^{30}$ avoided this simply by spreading a fresh monolayer over the solution after every forty or fifty dippings. This has already been recommended in the film deposition technique for obtaining a large number of monolayers. However, the collapse of stearate monolayers is very unlikely to occur. Otherwise their use in commercially available step gauges, (cf. Ref. 7) which are based on the known and controllable thicknesses of the monolayer, would be impossible.

\subsection{Film Structure}

The obvious choice for investigating detailed film structure is the well-known electron diffraction technique. Germer and Storks ${ }^{31}$, particularly, investigated the molecular arrangement of deposited mono- and multilayer films of fatty acids and their soaps using this powerful technique. In their studies in transmission as well as in reflection, by depositing films on thin supporting organic foils of Resoglaz and on a clean metal surface, the molecules were found to stand almost perpendicularly to the solid surface in the first monolayer. Barium stearate moleules had a more precise normal alignment than the stearic acid molecules. The hydrocarbon chains of stearate molecules in the first monolayer were close packed, but irregularly arranged on a clean metal surface, whereas regular arrangement was observed in layers on top of the first one in the case of films on Resoglaz foils. Thus, the layered structure of these stearate films was shown to form simple hexagonal crystals with the symmetry axis perpendicular to the film. The stearic acid molecules were found to form monoclinic crystals, with their chain axis nearly in the plane containing the surface normal and the dipping direction. In earlier studies ${ }^{5,6}$, it has been shown that these films consist of superposed sheets of oriented molecules, and form positive uniaxial birefringent crystals with the optic axis perpendicular to the plane of the film. However, some structural changes due to thermal disorientation of the molecules, or to evaporation were noticed at relatively high temperatures when the temperature was raised in electron diffraction studies $32-35$. This may be possible, because the soap multilayer films are relatively soft and have low melting points. On the other hand, in recent investigations by $\mathrm{Holt}^{29}$, the remarkable thermal stability of such films has been reported. In fact, these films could be heated at $90^{\circ} \mathrm{C}$ in damp air over prolonged periods (the melting point of stearic acid is $69^{\circ} \mathrm{C}$ ) without any damage. Further, Holt observed no change in the electrical properties even though the films were repeatedly and rapidly cycled from liquid nitrogen temperature at $50^{\circ} \mathrm{C}$ in air.

Based on the above structural information obtained by electron diffraction, these films were known to be monocrystalline in nature and thus were regarded as a special case of a layer-by-layer mechanical growth forming almost "two-dimensional" crystals. There is, however, evidence that barium behenate multilayers do, in fact, show an absence of crystallinity which has been demonstrated by electron micrographic studies ${ }^{36}$. It has also been reported by Knott et al. ${ }^{37}$ that some time may be required after multilayer formation for the formation of crystallinity, and no crystallinity was observed until 30-60 minutes passed. Marc and Messier ${ }^{38}$ have attributed the two absorption peaks, observed at $-100^{\circ} \mathrm{C}$ and $-30^{\circ} \mathrm{C}$, to dipole movements in the amorphous and crystalline regions of the behenate layers respectively. In fact, they had no clear cut evidence for amorphous zones in the monolayers and the qualitative interpretation offered by them was based merely on the assumption of disorganized structures. Therefore, the author would like to stress here that in future investigations one must be careful in the interpretation of the electrical data in terms of the film structure. Renewed structural investigations would also be desirable to make sure of the presence of amorphous regions in these films.

\subsection{Defect Nature}

Many of the earlier workers have reported that organic films obtained by the classical process of monolayer transfer always contained holes, cracks or other imperfections. For instance, Epstein ${ }^{39}$ demonstrated in his electron microscopic studies that the deposited films are discontinuous, and consist of clumps of molecules, when the transfer was accomplished at low surface pressures. Similarly, Ries and Kimball ${ }^{40}$ observed discontinuous circular "islands" or aggregates in the stearic acid monolayer transferred at $10 \mathrm{dyn} / \mathrm{cm}$. These observations were not surprising because the higher the surface pressure, the more 
compact will be the film formed. In fact the monolayers have been shown to be continuous and homogeneous at higher pressures ${ }^{41}$. Nevertheless, it would be an oversimplification to regard the film transferred at high surface pressure as perfectly uniform, coherent and defect free. Artifacts are introduced if proper care has not been taken either during the transfer process and the subsequent thermal evaporation of metal electrodes over the film may disturb the film structure. In some radioautographic studies, the presence of spots, striations and other irregularities such as "folded over" regions in the deposited barium stearate monolayer was demonstrated in the earlier work of Roberts and Gains $^{27}$. On the other hand, Handy and Scala ${ }^{42}$ also carried out radioautographic studies and have shown that these layers are generally uniform with no apparent gross defects. One reason for the presence of such defects in the films as demonstrated by these early workers is that the original technique of B-L had not then been perfected. Many of the recent modifications in this technique have made it possible to obtain films largely free from gross defects or imperfections.

The one study giving quantitative proof of the absence of any number of holes and conducting imperfections in such monolayers is that of Mann and $\mathrm{Kuhn}^{15}$. They had employed a modified B-L technique $^{23}$, and took other precautions. In their studies on breakdown phenomenon of Langmuir films (described later) Agarwal and Srivastava have also indirectly shown the absence of "weak spots", and thus they inferred that the films are free from gross defects. The development of striations in the deposited film was ascribed to the irregularity of the dipping and withdrawal motion of the substrate ${ }^{27}$. Recently, Nathoo ${ }^{19}$ has introduced a novel technique for this, and has succeeded in obtaining acceptable and reproducible devices for electrical measurements using this technique and the other precautions discussed above.

In spite of the fact that structural perfection may be achieved by using Langmuir mono- and multilayers, great attention must still be paid to identifying the defect structure of the films. Reliability and interpretation of electrical data very much depend upon the nature of the defects and therefore special care must be taken.

\subsection{Skeletonization Process}

It is an established fact that films of metal salts of fatty acids may contain free acid molecules, apparen- tly in the solid solution if the metallic ion concentration is low; their proportion depends upon the $\mathrm{pH}$ of the solution ${ }^{5,6}$. These free acid molecules can be dissolved out of the multilayers by soaking the film for a short time in benzene to which $1 \%$ of $90 \%$ ethyl alcohol is added ${ }^{20}$. This process is termed "skeletonization", and the film thus obtained is said to be "skeletonized"6, containing voids or holes in the places previously occupied by the acid molecules. Optical studies have shown that the films remain optically clear even after this treatment if the amount of acid molecules contained is not more than $60 \%$, but a striking change is observed in the interference colours reflected by the film. Further, it has been shown that this change in colour is due to a change of refractive index and not to a change of thickness. If half of the acid content is dissolved, there is found to be a change of less than $1 \%$ in the film thickness. The stability of such a skeleton film is not affected, as indicated by its extraordinary rigidity even when it contains up to $40 \%$ voids. The fact that the skeletonized film does not collapse was checked by Race and Reynolds ${ }^{43}$ who restored the original optical thickness by refilling the free spaces with some non-polar oil. In fact, these holes and voids in the skeleton films may be refilled with substances like petrolatum, or other hydrocarbons; subsequently, if required, these substances can be dissolved in benzene giving again the same skeletonized films. This method has already been used to obtain films with various refractive indices between 1.18 and $1.51^{20}$. However, not much attention has been paid to the study of the electrical behaviour of such skeleton films carrying foreign impurities like hydrocarbons. The author feels that the method is very attractive for obtaining doped-like organic dielectrics and further exploration of their electrical properties would be worthwhile. Race and Reynolds ${ }^{43}$ have made an attempt to study the electrical behaviour of such skeletonized films, and found the expected lowering of dielectric strength by skeletonization. The physical concept, that the remaining soap and air (or any hydrocarbon substituted) remain electrically in parallel, was found to hold true in their measurements.

As discussed above, the removal of free acid from the deposited films may also take place during their treatment in vacuum for elctrode evaporation. This process is known as "vacuum skeletonization". In fact, Roberts and Gains 27,28 have noticed removal of stearic acid from monolayers deposited on various substrates. Evidently, the presence of voids or holes as reported by many workers may be an effect of vacuum skeletonization. Many workers have failed to 
obtain useful devices for electrical measurements, and this is presumably because the spaces previously occupied by the free acid molecules may have been filled with the metal during thermal treatment in vacuum, thus shorting the device. Because of this, it is often stressed in the literature that Langmuir films must be treated carefully in vacuum, particularly when electrical measurements have to be made. However, great care must have been taken in the first place to hold the required $\mathrm{pH}$ of the solution, because the degree of conversion to soap depends upon the $\mathrm{pH}$. Since the soap or metal salts of long chain fatty acids are ionic substances forming stable monolayers (cf. Ref. 7, p. 193) it is possible that some ions are present in the deposited monolayer which may be transferred during deposition. It has been shown ${ }^{44}$ that with some cations, the ion composition of the deposited film is very different from that on the water surface, but much is unknown about the nature of such ions. The presence of such ions and their motion under an applied electrical field has recently been pointed out by Barraud and co-workers ${ }^{45}$ in their studies of thermally stimulated currents on MIM structures using Langmuir films.

\section{DIELECTRIC PROPERTIES}

Interest in the dielectric studies of Langmuir films of fatty acids and their metal salts grew rapidly in the period just after the advent of the B-L deposition technique. Such studies were taken up again only after a gap of more than two decades when it was realized that Langmuir films could be uniform in thickness, and act as good insulators. In this section the experimental measurements on resistance, capacitance and dielectric constant of these film systems are described. These measurements have been divided into two parts; the first describes the work carried out in the late forties and the second covers recent experimental work. The dielectric loss measurements and the theory for calculating the static dielectric constant are discussed.

\subsection{Earlier Measurements on Capacitance, Resistance and Dielectric Constant}

The earliest work of Porter and Wyman (PW) 46 reported measurements on the impedance of films and related phenomena which they carried out on films of $\mathrm{Cu}-\mathrm{Ba}$ stearate and $\mathrm{Ca}$ stearate using both $\mathrm{X}$ and $\mathrm{Y}$ type films. Initially, mercury droplets were used for small area probe measurements and an ac bridge was used for impedance measurements. The resistance of the films through the thickness was found to be very low $(<1 \Omega)$ with high signal voltages, whereas it was of the order of $M \Omega$ with signals of 1 or $2 \mathrm{~V}$. Measurements at frequencies of $1 \mathrm{MHz}$ and $0.244 \mathrm{MHz}$, and for films containing 7 to 141 monolayers gave dielectric constant values ranging between 1.9 and 3.5 with an average value of 2.5. Considerable variations in the results obtained on the same film with different drops, or with the same drop in different places, were due apparently to differences in the droplet size, or in the exact area of contact between the drop and film at different places. No significant difference was found in the capacitance or resistance values between $\mathrm{X}$ and $\mathrm{Y}$ films. In both types of films the capacity decreased with thickness, as was expected from the relation used by them for capacity determination, i.e.

$$
C=A \epsilon / 4 \pi N d,
$$

in which $\epsilon$ denotes the dielectric constant, $N$ the number of layers, $d$ the layer thickness and $A$ the area of contact between drop and film. On the other hand, the values of the resistance per layer showed a definite increase with the thickness of the film. The specific resistance of films, thus determined from their values of the resistance per layer, was about $10^{13} \Omega / \mathrm{cm}^{2}$. Some dc resistance measurements were also made by them using a drop of tap water in place of a $\mathrm{Hg}$ drop and a striking difference between the results was found. With mercury drops the film ruptured permanently when a breakdown voltage of $10^{6} \mathrm{~V} \mathrm{~cm}^{-1}$ was exceeded and the resistance was low. No such rupture was observed with water drops. It was concluded that the different affinities of the two liquids for the film surface may have been responsible for the observed difference, and that the water molecules were probably able to penetrate the films. Another conclusion arrived at was evidence of power absorption in the films, increasing with frequency over the range studied. This was concluded because the resistance of the films measured at both frequencies ( 1 and $0.244 \mathrm{MHz}$ ) was far less than that determined from their dc measurements.

Race and Reynolds (RR) ${ }^{4}$ in 1935 made some preliminary attempts to measure the dielectric properties of barium stearate films, and found some experimental limitations. For instance, the bridge equipment used was not sensitive; the area of contact of mercury droplets was not accurately measured and dust contamination could not be avoided. Nevertheless these results encouraged them to develop a sophisticated experimental set-up and the necessary bridge equipment ${ }^{47}$. Multilayer films were deposited 
on a clean polished chromium slide and mercury droplets were used as the counter-electrode. All the measurements were made over a frequency range from 40 to $10^{6} \mathrm{~Hz}$ using comparatively thick multilayers (of both $\mathrm{X}$ and $\mathrm{Y}$ types) of various stearates and arachidates. The results obtained by them were:

1) The dielectric constant of various multilayers was $\cong 2.55$, with standard variation of $3 \%$. The variation was attributed to the $\mathrm{pH}$ of the solution and therefore the degree of conversion of acid to soap.

2) The dielectric constant was found to be independent of thickness as well as of frequency in the range studied.

Further, these workers ${ }^{43}$ measured the dielectric parameters of films which were soaked for from one to ten seconds in a solvent such as benzene. The benzene dissolved the free acid leaving the soap film as a skeleton with air filling the spaces previously occupied by the acid. For calculating the dielectric constant of such a skeletonized film, the remaining soap and the air spaces were considered in parallel. The relation used was

$$
\epsilon_{1}=\epsilon_{0}\left(\frac{d_{1}}{d_{0}}\right)+\epsilon_{a}\left(1-\frac{d_{1}}{d_{0}}\right)
$$

where $\epsilon_{0}$ and $\epsilon_{1}$ are respective dielectric constants of the unskeletonized and skeletonized film; $\epsilon_{a}=1$ is that of air and $d_{1} / d_{0}$ is the ratio of the densities of the skeletonized and unskeletonized films. Their results on unskeletonized (0) and skeletonized (1) films of cadmium arachidate are given in Table I. In the table, $t_{0}$ and $t_{1}$ refer to the apparent optical thicknesses before and after skeletonization as determined by Blodget $t^{48}$. To check the collapse behaviour of the skeletonized films, the air space was refilled by allowing a drop of oil to run over the film, and the dielectric constant was measured again and calculated using Eq. (4.2) by substituting the dielectric constant of oil for $\epsilon_{a}$. RR used non-polar oil as well as a polar liquid (tetrachlorodiphenyl) to refill the air spaces in the skeleton. The corresponding values of the dielectric constant have been represented by $\epsilon_{2}$ and $\epsilon_{3}$, respectively, in Table I.

As is evident from Table $I$, the agreement between measured and calculated values of $\epsilon_{1}$ is sufficiently close. Obviously, the dielectric constant of the film $\left(\epsilon_{2}\right)$ increased as expected, when the air spaces in the skeletonized film were refilled with a non-polar oil (columns 8 and 9) having a dielectric constant of 2.15. This was interpreted as a check on the structural conception of a skeletonized film, i.e. the assumption that the remaining soap and air are electrically in parallel (columns 6 and 7). However,

TABLE I

Electrical data on unskeletonized (0) and skeletonized (1) films of cadmium arachidate. [After Race and Reynolds ${ }^{4}{ }^{7}$.]

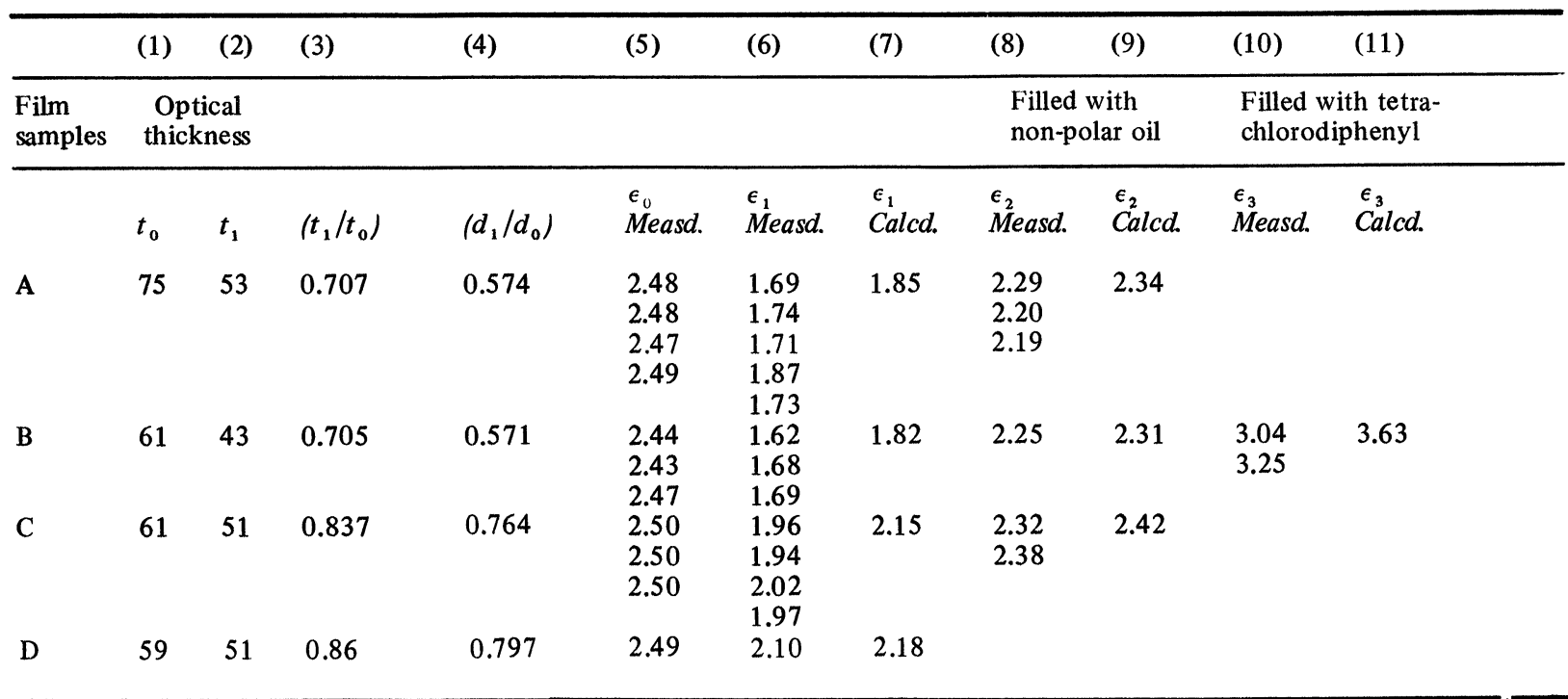


considerably higher calculated values of $\epsilon_{3}$ than the measured one (columns 10 and 11) are found with a polar liquid (dielectric constant 5.22), which were interpreted as indicating a reduction of the polar orientation of the liquid within the matrix of cadmium arachidate molecules.

Buckwald, Zahl and co-workers ${ }^{49,50}$ also became interested in measurements of the resistance and capacity of alkali stearates consisting of varying numbers of layers, and deposited on metal surfaces. Preliminary measurements were made on barium stearate films ${ }^{49}$, and later calcium stearate films. A special cell was designed, consisting of a cylindrical glass vessel partially filled with calcium chloride solution. Two metal electrodes were immersed in this solution and the films to be studied were deposited directly at the electrode surfaces. Using a slightly modified conventional ac Wheatstone bridge network, a number of readings for films (1-41 layers) indicated the decrease in capacitance with increasing thickness for one set of their measurements. However, in another set of measurements, an increase to a maximum of three layers was observed after which capacitance started decreasing. The latter data has been attributed to the presence of serious imperfections in the films. An average value of the dielectric constant $(\cong 3.7)$ for 41 layers was thus calculated from the observed capacitance values $\left(\cong 10^{-6} \mathrm{~F}\right)$. Two-fold variation was found in the apparent dielectric constant, and several orders of magnitude variation in the resistance in the thickness range studied. Further, both the capacitance and resistance were found to be dependent on the nature and concentration of the electrolyte. From the studies using copper sulphate as an electrolyte of varying concentrations, they inferred that the significant variation of the electrical (and presumably the structural) properties of the film may be due to the presence of the copper ion, because these values differed widely from those taken with $\mathrm{Ca}_{2} \mathrm{Cl}_{2}$ as an electrolyte. Finally, these workers emphasised that the stearate films may be electrically modified by simply varying the water content in the electrolytic solution. It was further stated that the mercury droplet method of $\mathrm{RR}^{43}$ has many advantages over that used in their own work.

\subsection{Recent Measurements on Capacitance, Resistance and Dielectric Constant}

Handy and Scala ${ }^{42}$ (HS) after more than two decades realized that mono-molecular layers obtained by the B-L technique satisfy all the conditions required for reliable electrical measurements. For instance, such organic layers are thin, homogeneous, uniform in thickness and excellent insulators. In their detailed investigations, they tried to obtain a more compact film to minimize the presence of voids or holes in the organic multilayer, and used evaporated metal electrodes. Figure 3 shows the results of capacity measure-

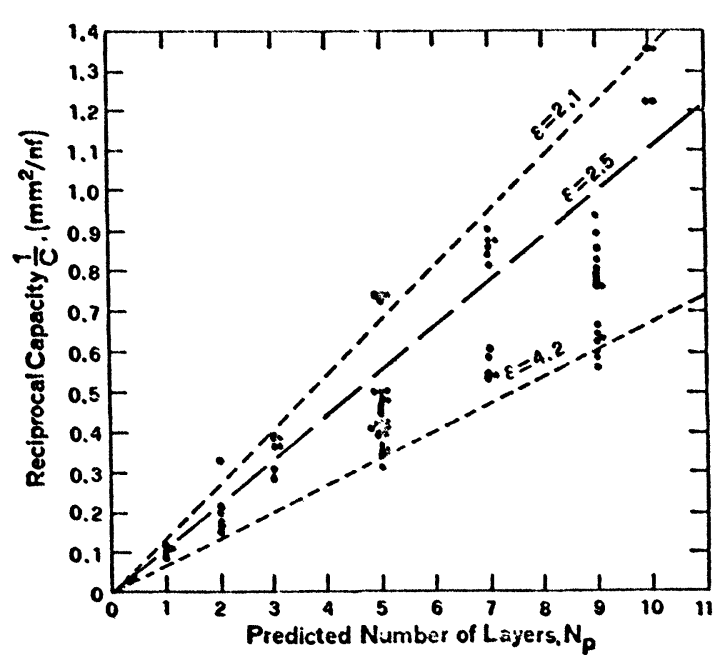

FIGURE 3 Reciprocal capacity $1 / C$ vs. predicted number of layers $N_{p}$. The heavy dashed line corresponds to the bulk value $(\epsilon=2.5)$ and the dotted lines represent the experimental values of $\epsilon=2.1$ and 4.2. The cluster of points shown in the figure fall on top of each other and are slightly misplotted to the left or right to show the density distribution of data. [After Handy and Scala ${ }^{42}$.]

ments on some 75 samples in which reciprocal capacity $(1 / C)$ is plotted as a function of predicted number of layers $\left(N_{p}\right)$. The capacitance measurements thus performed on stearate films (1-10 layers) led to $\epsilon$ values between 2.1 to 4.2 with a bulk value of $\epsilon=2.5$. These results agreed well with those obtained by PW using liquid contacts. Figure 4 shows a typical graph of capacity as a function of frequency (uppercurve) over the frequency range $0.1-20 \mathrm{KHz}$. On the basis of this curve, HS predicted a slight dependence of $\epsilon$ on frequency (a drop of about $5 \%$ over the range studied). The lower curve of Figure 4 shows the variation of the imaginary part of complex dielectric constant (proportional to $C \times D, D$ being the dissipation factor) with frequency. Evidently, there is a slight maximum near $700 \mathrm{~Hz}$ which has been taken to be indicative of the presence of a weak polar absorption mechanism with a 
characteristic relaxation time $(1 / 2 \pi \mathrm{fm})$ of $0.23 \mathrm{~m} \mathrm{sec}$. The measurement uncertainty in the dissipation factor $D$ becomes comparable to the observed values towards the high frequency region $(>10 \mathrm{KHz}$ ) (see the lower curve of Figure 4).

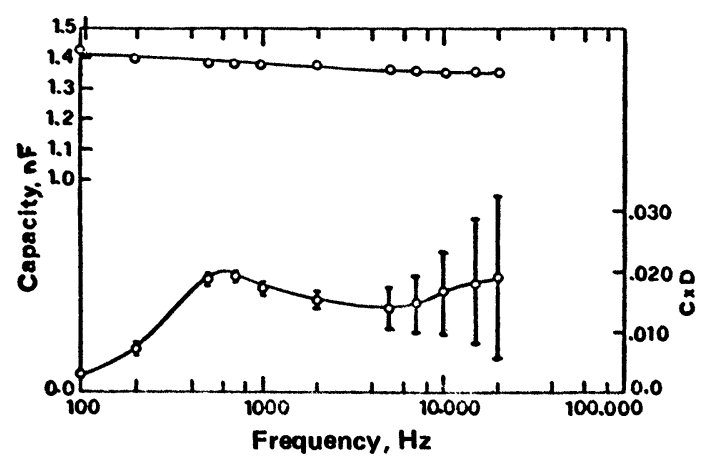

FIGURE 4 Capacity $C$ vs. frequency $f$ (upper curve) and capacity $\mathrm{x}$ dissipation factor (CXD) vs. frequency $f$ (lower curve). [After Handy and Scala ${ }^{4}$.]

The contribution of the oxide layer formed between the lower metal electrode and the organic film was neglected in presenting capacity measurements in view of its small resistivity compared with that of the organic layers measured. In the ohmic conduction region $(\ll 50 \mathrm{mV})$, a wide range of variation of resistivity for the same film thickness was observed which supported the contention of a porous structure in these films. $\mathrm{HS}^{42}$ thus concluded that the resistivity obtained for smaller thicknesses is characteristic of the oxide film, and its upper limit for a given film thickness corresponds to the film possessing the smallest fraction of voids. The important conclusions arrived at were that the poor reproducibility of the characteristics is due to voids and inhomogeneities in the film, and a reactive and hence oxidized surface is necessary for the formation and retention of low porosity layers. HS also found that the physicochemical nature of the substrate influenced the adhesion of more than the first monolayer, and that the porosity alone was considered unlikely for such long-range effects. Positive ion adsorption, as described by Goranson and Zisman $^{51}$, has been suggested as a possible alternative and was shown to be consistent with some aspects of the electrical measurements. Beck ${ }^{52}$ has reported measurements on capacitance for fatty acid monolayers of different chain lengths sandwiched between A1 electrodes, and found capacitances reproducible to within $3 \%$, unlike HS who found a variation of $50 \%$ in capacitance.

Drexhage and $\mathrm{Kuhn}^{53}$ have measured the capacitance of cadmium arachidate and cadmium stearate films sandwiched between aluminium electrodes. Astonishingly, they found this method suitable for the determination of film thickness even for a single monolayer, the capacitance $C$ being given by Eq. (4.1). As expected, a linear dependence of $1 / C$ with the number of monolayers $(N)$ was found. Evidently this means that $\epsilon$ is independent of thickness. The slopes of the straight lines in a plot $1 / C$ vs $\mathrm{N}$ yielded a value of $d=26.6 \AA$ for cadmium stearate by using $\epsilon=2.40$ (obtained simply by the square of the refractive index of barium stearate as determined earlier ${ }^{6}$ ). Holt ${ }^{29}$, using Langmuir monoand multilayer films as thin film dielectrics ${ }^{54}$, carried out preliminary measurements on stearic acid and stearate films sandwiched between metal electrodes. The standard deviation of $C / A$ in each device was as low as $3 \%$, which he suggested to be of the order of accuracy of producing and measuring the areas of the base and counter-electrodes. No significant variation of $C$ and hence $\epsilon$ with frequency were found. The interesting properties thus predicted by Holt, i.e. thermal stability and uniformity of organic films, have already been discussed. Another important suggestion made by him is that the dielectric constant can be varied by altering the metal stearate proportions in the multi-monolayers.

In many of the measurements discussed above, the organic film was sandwiched between evaporated aluminium electrodes. The fact that a natural oxide layer grows on the base aluminium electrode was realized, but its effect on the capacitance values of the device was neglected ${ }^{42}$, since the resistivity of the oxide film is small compared with the resistivity of the organic layers. In the work of Mann and Kuhn $(\mathrm{MK})^{15}$, the presence of such a thin oxide layer between metal electrodes and fatty acids is considered; the relation used by them is

$$
\frac{1}{C}=\left(\epsilon_{a} A\right)^{-1}\left(\frac{d_{\mathrm{ox}}}{\epsilon_{\mathrm{ox}}}+\frac{N d}{\epsilon}\right)
$$

where $d_{\mathrm{ox}}$ and $\epsilon_{\mathrm{ox}}$ are the thickness and dielectric constant of the oxide layer, $\epsilon_{a}$ is a dielectric constant of air, and all the other symbols have the usual meaning. For calculating $\epsilon$ values, they plotted $1 / C$ as a function of superimposed layers $(N)$ and, from the slope of straight lines thus obtained, the $d / \epsilon$ values were determined. A plot of $1 / C$ vs $N$ is given in 


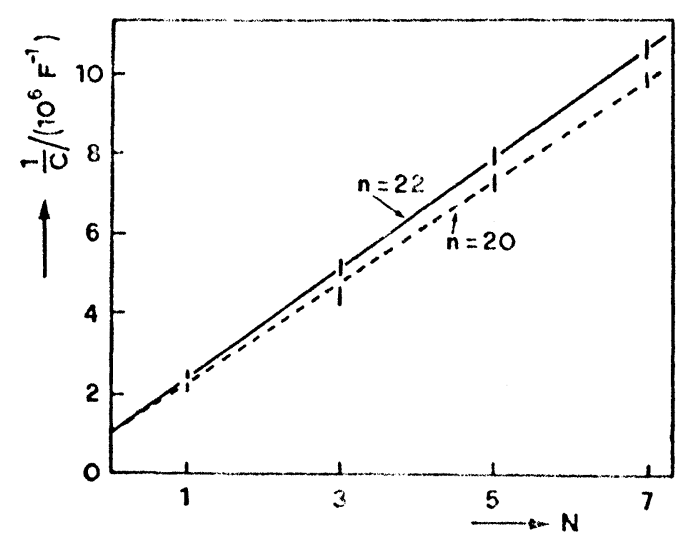

FIGURE 5 Reciprocal capacity $1 / \mathrm{C}$ vs. number of layers $\mathrm{N}$ for cadmium stearate $(n=20)$ and cadmium behenate $(n=22)$. [After Mann and Kuhn ${ }^{15}$.]

Figure 5 for stearate and behenate films which shows the same y intercept in both cases. The capacitance, and hence the $\epsilon$ values were determined by $\mathrm{MK}^{15}$ for a series of fatty acid monolayers of Cd salts. For instance, the values for stearic acid $\epsilon=2.71 \pm 0.17$ and for arachidic acid $\epsilon=2.52 \pm 0.07$ determined by them were found to be comparable with respective values $\epsilon=2.59 \pm 0.08$ and $\epsilon=2.49 \pm 0.06$ determined earlier by $R^{4}{ }^{4}$. However, their calculations ${ }^{15}$ show an increasing $\epsilon$ with decreasing chain length, which is qualitatively explainable due to the influence of the highly polarizable cadmium carboxylate groups, which increases with decreasing fatty acid chain length.

Recent measurements of capacitance by Leger et al. ${ }^{55}$ have also shown a linear dependence of $1 / C$ with respect to the number of transferred monolayers. They made use of this simple method for measuring the thicknesses of organic films and found that it yielded satisfactory results. A change of less than $10 \%$ in the capacitance of stearic acid films, increasing slightly towards the low-frequency region, has been reported in the frequency region $10^{-4}$ to $100 \mathrm{~Hz}$ by Nathoo ${ }^{19}$.

More systematic investigation have recently been made by Khanna and Srivastava ${ }^{56}$ (KS) on the capacitance and hence dielectric constant of a few fatty acid salts. In their measurements, sandwich structures of the type A1-film-A1 were employed and the capacity values were determined initially without considering the effect of the oxide layer ${ }^{56}$. For all the film systems, measurements were made on 8 and 110 layers using a universal bridge. The capacitance as well as the dielectric constant values were found to be distinctly different in the two cases. However, all these results were in good agreement with their theoretically calculated values of $\epsilon$. The values obtained by KS, experimentally as well as theoretically, are tabulated in Table II. A slight variation in these values arises because their theoretical formulation $^{57,58}$ is based on an ideal structure of the film which is difficult to achieve in practice. Subsequently, Khanna ${ }^{59}$ calculated the $\epsilon$ values considering the effect of the oxide layer, because this can be electrically insulating and can contribute significantly to the capacitance of the sandwich structure. For this, the expression already employed by Mann and Kuhn. Eq. (4.3), was used, and the values for $d_{\mathrm{ox}}=30 \AA$ and $\epsilon_{\mathrm{ox}}=8$ were taken. The $\epsilon$ values thus obtained have also been given in Table II. Evidently, the effect of the oxide layer is more significant in the case of a monolayer as compared with that for multilayers.

To explain the significant difference of $\epsilon$ values for monolayers and multilayers obtained theoretically as well as experimentally, Khanna ${ }^{59}$ carried out a

TABLE II

Calculated and measured values of dielectric constant of a few long chain fatty acid Langmuir films. [After Khanna and Srivastava ${ }^{56,59}$.]
(1)
(2)
(3)
(4)
(5)
(6)

\begin{tabular}{|c|c|c|c|c|c|c|c|c|c|}
\hline film substance & $\begin{array}{l}\text { monolayer } \\
\text { thickness }\end{array}$ & \multicolumn{2}{|c|}{$\epsilon_{\text {calc. (from theory) }}$} & \multicolumn{2}{|c|}{$\begin{array}{l}\epsilon_{\text {calc. (from experimental }} \\
\text { data when oxide layer } \\
\text { effect was inclusive). }\end{array}$} & \multicolumn{2}{|c|}{$\begin{array}{l}\epsilon_{\text {calc. }} \text { (when oxide } \\
\text { layer effect excluded). }\end{array}$} & \multicolumn{2}{|c|}{$\begin{array}{l}\text { Capacitance per unit } \\
\text { area } \mu \mathrm{F} / \mathrm{cm}^{2}\end{array}$} \\
\hline Ba-palmitate & 23.25 & 1.65 & 2.90 & $1.67 \pm 0.12$ & $2.85 \pm 0.20$ & 2.29 & 2.88 & 0.636 & 0.00988 \\
\hline Ba-behenate & 30.05 & 1.50 & 2.00 & $1.60 \pm 0.10$ & $2.25 \pm 0.02$ & 2.01 & 2.27 & 0.470 & 0.00604 \\
\hline
\end{tabular}


systematic and detailed study of the thickness dependence of the dielectric constant from 1 to 80 monolayers. It was observed that the dielectric constant increased slowly with thickness initially, but saturated at a particular thickness $(\cong 1000 \AA)$ for all types of Langmuir films. A typical graph is shown in Figure 6, in which the dielectric constant $\epsilon$ is plotted as a function of film thickness $d$ for barium stearate films.

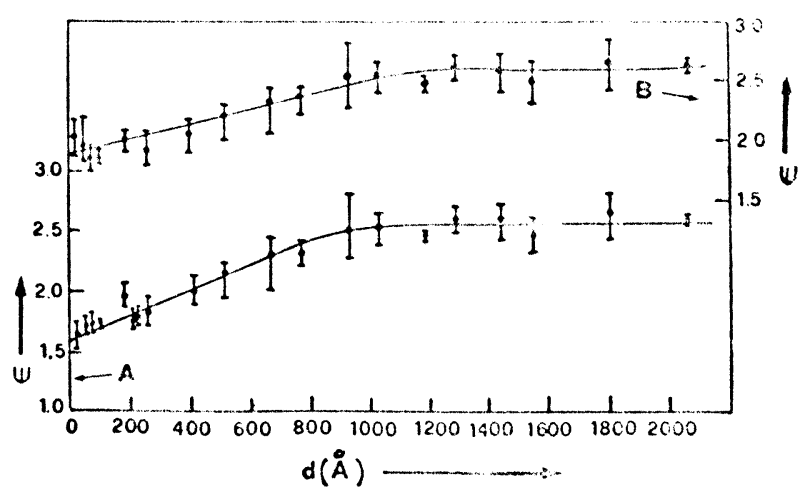

FIGURE 6 Dielectric constant $\epsilon$ vs. film thickness $d$ of barium stearate film. (A) without oxide layer effect, (B) with oxide layer effect. [After Khanna ${ }^{59}$.]

The upper curve (B) represents the variation of $\epsilon$ when oxide layer effects were taken into account, and the lower curve (A) shows when no such effects were considered. This unusual behaviour has not been reported in the earlier works, and the constancy of the dielectric constant with respect to thickness has been shown $15,42,53,55$. However, Khanna ${ }^{59}$ has explained her results qualitatively as given below. The observed dependence has been attributed to the porosity of films in the lower thickness range which decreases with increasing thickness. In fact, an exactly similar thickness dependence of the dielectric constant has been observed in evaporated $\mathrm{ZnS}$ films ${ }^{60}$, which has been interpreted in terms of decreasing porosity or increasing continuity of films with increasing thickness. The saturation of the dielectric constant at higher thickness ranges has also been observed for evaporated $\mathrm{ZnS}$ films. To explain why other workers failed to obtain such a dependence, it has been suggested that the films obtained by them were more compact. For example, in the present work, the film transfer was accomplished at a surface pressure $\cong 30 \mathrm{dyn} / \mathrm{cm}$, whereas in the work of Handy and $\mathrm{Scala}^{42}$, a high surface pressure of $39 \mathrm{dyn} / \mathrm{cm}$ was used. Ries et al. ${ }^{61,62}$ have already shown that voids in the monolayer are inevitable if the transfer is accomplished at a surface pressure significantly below the collapse pressure $(42 \mathrm{dyn} / \mathrm{cm}$ in the case of stearic acid films). Further, the thickness of about $1000 \AA$ at which the $\epsilon$ value saturates has been predicted as a rough estimate of the range of electrostatic interaction in Langmuir fatty acid soaps.

The author feels, however, that the explanation offered above is in no way sufficient to explain this unusual behaviour of the dielectric constant. Presumably the influence of highly polarizable metal carboxylate groups, which increases with increasing volume ratio, would be a better explanation for increasing $\epsilon$ with decreasing chain length for a given number of layers ${ }^{15}$. For $\epsilon$ saturating after first increasing with thickness, the qualitative explanation may be that dielectric losses are significantly less at higher film thicknesses, and therefore the dielectric constant does not change. The magnitude of dielectric losses has been related to the compactness of the monolayers in recent measurements of Marc and Messier ${ }^{38}$, and it has been shown that there is a decrease with increasing compactness. Nevertheless, rigorous experimental work is required to investigate the rôle of structural defects like voids, holes or inhomogeneities on the dielectric constant of Langmuir films. Also, the observed increase of $\epsilon$ may be understandable in terms of the increasing polarization with increasing number of monolayers. This, in fact, has already been shown by Tanguy and Hesto experimentally ${ }^{63}$, but it requires some structural changes inside the film which, in itself, is a problem yet to be studied.

\subsection{Theoretical Work}

Unfortunately, not much attention has been paid to developing the theoretical formulation of dielectric parameters of Langmuir films. The only attempt in this direction has been due to Khanna and coworkers ${ }^{57,58}$ who developed a theory for calculating the static dielectric constant of Langmuir films. Their theory is based on the layered structure of Langmuir films constituting a simple hexagonal array, with the molecules oriented normal to the supporting surface and separated by a distance $4.85 \AA^{31}{ }^{31}$ Müller's assumption for calculating the Lorentz field for a simple hexagonal lattice ${ }^{64}$ could not be applied here because the molecular size $(\cong 25 \AA)$ is large compared with the lateral intermolecular distance $(4.85 \AA)$. The local field has been calculated by summing up the externally applied field and the field due to all other dipoles within the specimen. A reasonable assumption 
made for this purpose is that each molecule is divided into small $\mathrm{CH}_{2}$ groups separated by $1.27 \AA$ along the chain axis (and, of course, the end carboxylate group). Further, all the dipoles are assumed to be point dipoles, parallel to each other, with their axis taken to be the external field direction ( $\mathrm{Z}$ axis)

\subsubsection{Monomolecular films Following Salem's} assumption ${ }^{65}$ that $D$, the mutual distance between two parallel linear chains, can be as small as about $4 \AA$ if the basic units (in the present calculations, $\mathrm{CH}_{2}$ groups are the basic units) are bonds of average length $1.5 \AA$, an expression for the contribution due to intermolecular interaction between two molecular hydrocarbon chains was obtained ${ }^{57}$;

$$
E_{1}^{z}=p\left[-\frac{N}{D^{3}}+2 \sum_{\mathrm{x}=1}^{\mathrm{N}} \frac{2 \lambda^{2} X^{2}-D^{2}}{\left(D^{2}+\lambda^{2} X^{2}\right)^{5 / 2}}(N-x)\right],
$$

where $p$ is the moment of the basic $\mathrm{CH}_{2}$ unit $\left(p=\alpha E_{10 c}\right), \alpha$ is the electronic polarizability of the $\mathrm{CH}_{2}$ group $\left(1.84 \times 10^{-24} \mathrm{~cm}^{3}\right.$, cf. Ref. 66), $\lambda$ is the distance between the two $\mathrm{CH}_{2}$ groups along the chain axis $(1.27 \AA)$ and $N$ is the number of $\mathrm{CH}_{2}$ groups along the molecular chain. The electronic polarizability here is assumed to be isotropic, and the two chains of fatty soap molecule are considered to be coincident. For calculating the effect of the end carboxylate group (unit), the relation derived was

$$
\begin{aligned}
E_{1(\mathrm{end})}^{z}=p_{\mathrm{end}}\left[-\frac{1}{D^{3}}\right. & \left.+\sum_{i} \frac{2 X_{i}^{2}-D^{2}}{\left(D^{2}+X_{i}^{2}\right)^{5 / 2}}\right] \\
& +p \sum_{i} \frac{2 X_{i}^{2}-D^{2}}{\left(D^{2}+X_{i}^{2}\right)^{5 / 2}}
\end{aligned}
$$

where $p_{\text {end }}$ is the moment of the end unit and $X_{\mathrm{i}}$ is the distance of the $i^{\text {th }} \mathrm{CH}_{2}$ unit from the end unit along the $Z$ direction.

For calculating the intramolecular contribution to the local field, the interaction between the units of the same molecule was calculated using the expression

$$
E_{2}^{z}=\sum_{i} \frac{2 p_{i}}{Z_{i}^{3}}
$$

where $p_{i}$ is the moment of the $i^{t h}$ unit and $Z_{i}$ is the distance between the reference and the different unit along the $Z$ direction.
4.3.2 Multimolecular films In the case of multimolecular films, the molecules are considered to be continuously distributed in all the layers except in the one in which the reference molecule is situated. Thus, following Müller's treatment ${ }^{64}$ for layer lattices. the contribution of the continuous distribution to the local field is $4 \pi P,(P$ is the polarization) and that of the reference layer is that calculated for a monolayer the one by the above method. The density of soap films is taken here to be equal to that of the bulk which is, however, not a very good approximation.

For calculating the dipole moment, the total polarizability has been taken as the sum of electronic and atomic polarizabilities of all the groups, and the orientational polarizabilities of the polar end group. However, the atomic polarizabilities, being small, are neglected. The contribution due to metallic ion presence in the end group has also been neglected in the calculation of orientational polarizabilities, and the end group is assumed equivalent to the $-\mathrm{COOH}$ polar group, its dipole moment being $1.8 \mathrm{D}$ (cf. Ref. 67). Finally, the static dielectric constant $\epsilon_{\mathrm{s}}$ along the field direction (the symmetry hexagonal axis), which is one of the principal axes of the dielectric tensor, is calculated using

$$
\epsilon_{\mathrm{s}}=1+\frac{4 \pi P}{E}
$$

Using this theoretical approach, Khanna and co-workers first calculated $\epsilon_{s}$ for barium stearate mono- and multilayers and found these values to be 1.6 and 3.0, respectively. ${ }^{57}$ Later they reported corrected values of $\epsilon_{s}$ for barium stearate as 1.7 and 3.1 , respectively, for a monolayer and multilayer. ${ }^{58}$ Here another assumption was introduced that a barium stearate molecule is equivalent to two "effective molecules", each consisting of one hydrocarbon chain and half of the end carboxylate group. These "effective molecules" were thought to form a hexagonal array with their axes normal to the supporting surface. Their previous assumption ${ }^{57}$ that two hydrocarbon chains are coincident was not consistent with the film structure reported by Germer and Storks ${ }^{31}$. Further calculations of $\epsilon_{s}$ for a few other fatty acid soaps were carried out ${ }^{56}$ and these are given in Table II. All these calculations are done only up to seven nearest neighbours, and short-range interactions, being small, have been neglected. The surface effects have already been considered in the calculations on monomolecular films, and these are assumed to be insignificant for multimolecular (sufficiently thick) films. 
It is evident from the above review of dielectric properties that our knowledge about them has advanced somewhat through experimental measurements, but theoretical understanding is still lacking. It is therefore necessary to attach special attention to more rigorous theoretical work. The theoretical formulation of Khanna et al. 57,58 described above has been based on a simple classical approach, and a number of the assumptions made do not hold under actual experimental conditions. For example, Müller's treatment for layer lattices, which has been followed for multimolecular films, no longer holds if the macroscopic volume of the sphere is large enough to permit the molecules to remain embedded in a continuous medium with the same dielectric constant. The interaction of the sphere with its surroundings thus requires electrostatic calculation which should be included in future work. Metal ions such as $\mathrm{Ca}^{++}$have been shown to reduce the mobility of the polar groups by linking one molecule with another ${ }^{38}$. The contribution of such ions in the calculation of electronic polarizability of the end carboxylate group [such as $(\mathrm{COO})_{2} \mathrm{Ba}$ ] has been neglected in the above calculations. Many more assumptions in the approach of Khanna and co-workers require attention. More interesting would be the extension of this work to time-dependent fields, which obviously would involve the complex dielectric constant of the system, and $\epsilon_{s}$ would become a special case of such a formulation. Both the real and imaginary parts of the complex dielectric constant would be frequency-dependent, and the imaginary part would be of more relevance to account for the dielectric losses. A quantummechanical approach would be even more difficult, and a description is beyond the scope of the present paper. For interested workers, reference should be made to the monograph by Frohlich ${ }^{68}$, which is a comprehensive treatise on the theory of dielectrics.

\subsection{Dielectric Loss Measurements}

Some preliminary dielectric loss measurements were carried out in the late forties by $R R .^{43}$ They found that the dielectric loss neither depends upon the frequency, in the range from 40 to $10^{6} \mathrm{~Hz}$, nor is it affected by the film constitution. The dielectric losses thus determined by RR were low, their order of magnitude being lower by a factor of $10^{-3}$ than that obtained by Buckwald et al. ${ }^{49}$ Similarly, Holt ${ }^{29}$ also obtained some information that the tangent loss angle $(\tan \delta)$ drops from 0.02 at $225 \AA$ to 0.006 at $3700 \AA$. The variation of $\tan \delta$ with frequency was predicted to follow normal thin film behaviour. However, these measurements did not furnish any useful information except the order of magnitude of $\tan \delta$, since the standard deviation was $25 \%$.

A systematic study of dielectric losses in Langmuir films of calcium stearate and behenate was reported recently by Marc and Messier ${ }^{38}$ (MM). The structure used was $\mathrm{A} 1-\mathrm{film}-\mathrm{A} 1$ and the measurements were performed in the temperature range $\left(-170^{\circ} \mathrm{C}\right.$ to $\left.+50^{\circ} \mathrm{C}\right)$ and over the frequency region $(100 \mathrm{~Hz}-50 \mathrm{KHz})$. All measurements were made after 24 hours degassing under vacuum, since the dielectric losses decreased rapidly by a factor of 2 during the first few hours of degassing. The results thus obtained were analysed by means of the equivalent circuit shown in Figure 7, where $C_{m}$ and $R_{m}$ refer to the

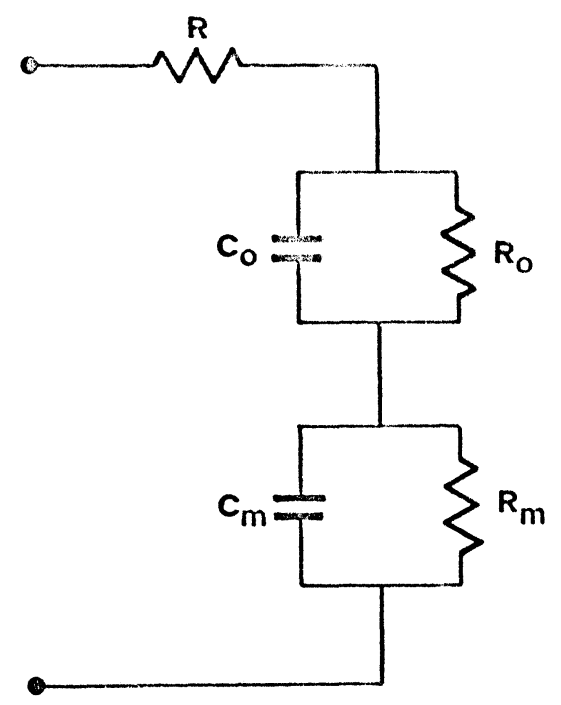

FIGURE 7 Equivalent electrical circuit of a metalLangmuir film-metal structures. [After Marc and Messier ${ }^{33}$.]

capacitance/area and resistance of the organic monolayer, and $C_{0}$ and $R_{0}$ to that of the aluminium oxide layer, respectively. $\mathrm{R}$ is the series resistance of several ohms due to the metal electrodes, which introduce series losses of the form $2 \pi \mathrm{Rf} ; f$ denoting the frequency. Since the loss angle of the structure ( $\tan$ $\delta$ ) varied as $f$ at high frequencies, they subtracted the series losses $\cong R C \omega$ and considered the following quantity:

$$
P_{s}=\frac{\tan \delta-R C \omega}{C}=\frac{1}{\omega R_{0} C_{0}{ }^{2}}+\frac{1}{\omega R_{\mathrm{m}} C_{\mathrm{m}}{ }^{2}},
$$

where $C=\left(C_{0}+C_{m}\right)^{-1}$. 
Evidently the quantity $P_{s}$ is proportional to the losses per unit area in the alumina and the monolayers. Further, it was assumed that all $N$ monolayers are equivalent, and therefore can be represented by $N$ impedances in series. Calling $d_{m}$ the thickness, $\epsilon_{m}^{r}$ the relative dielectric constant and $\rho_{m}$ the resistivity of each of the monolayers, $P_{s}$ may be given by

$$
P_{s}=\frac{1}{\omega R_{0} C_{0}{ }^{2}}+\frac{1}{\omega \epsilon^{2}} \cdot \frac{d_{m}}{\left(\epsilon_{m}^{r}\right)^{2} \rho_{m}} \cdot N .
$$

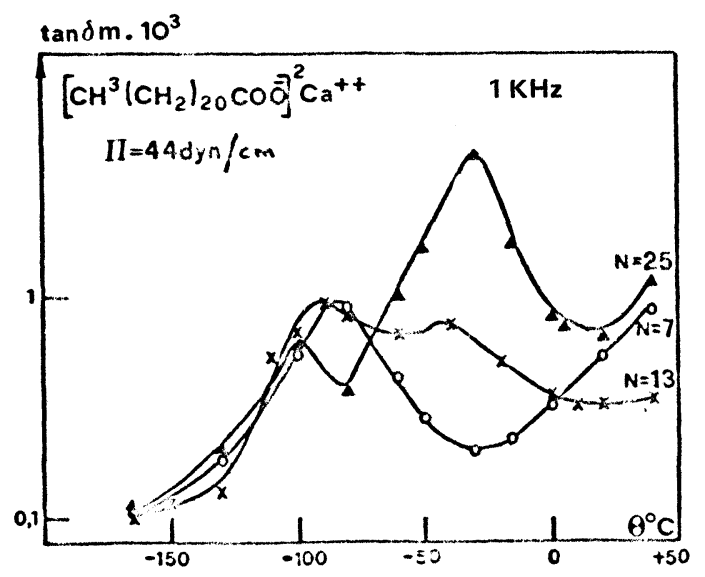

FIGURE 8 Loss angle of behenate layers $\tan \delta_{\mathrm{m}}$ vs. temperature $\theta$ measured at $\mathrm{f}=1 \mathrm{KHz}$ and at a surface pressure $\pi$ of $44 \mathrm{dyn} / \mathrm{cm}$. [After Marc and Messier ${ }^{38}$.]

Thus $P_{s}$ depends linearly on $N$. Figure 8 shows the variation of the loss angle of a behenate film $\left(\tan \delta_{m}\right)$ measured at $1 \mathrm{KHz}$ as a function of temperature. The curves have been plotted for films consisting of 7,13 and 25 monolayers which were transferred at a surface pressure of $44 \mathrm{dyn} / \mathrm{cm}$. Evidently, two absorption peaks are observed around $-100^{\circ} \mathrm{C}$ and $-30^{\circ} \mathrm{C}$. The peak around $-100^{\circ} \mathrm{C}$ is present in all three films studied, whereas the other appears only in thick structures $(N=13$ and 25$)$ which are known to crystallize more easily. In a separate measurement, they found that the latter peak disappears when the structure of the film is disorganized (see curve $b$ of Figure 9). Curves (a) and (b) in Figure 9 represent dielectric loss plotted against temperature measured at two different points of the same film. Curve (a) corresponds to a point in the middle of the film where the molecules are well organized, and curve (b) to a point near the edge of the substrate where the layer might get disturbed due to dipping or withdrawal.

The results obtained in the work of MN are summarized below:

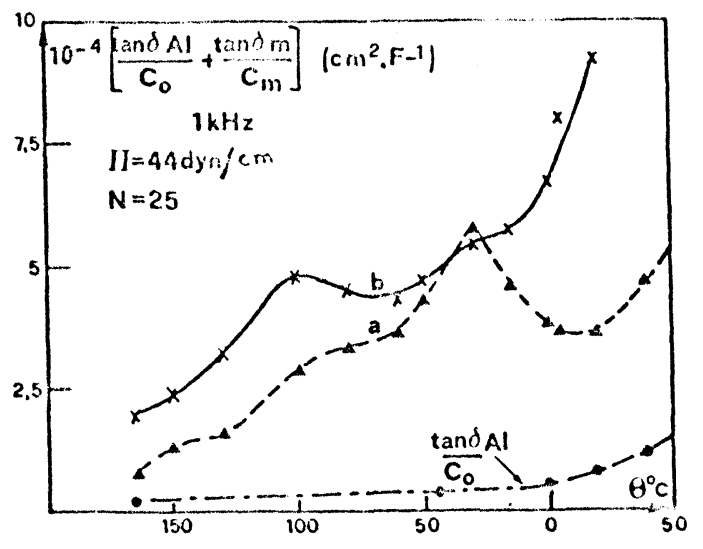

FIGURE 9 Comparison of dielectric losses with respect to temperature (a) corresponding to a point in the middle of the behenate film, well organized structure, and (b) at a point near the edge, disorganized structure. Data reported for $N=25, \pi=44 \mathrm{dyn} / \mathrm{cm}$ and $\mathrm{f}=1 \mathrm{KHz}$. The lowest curve represents losses due to oxide layer. [After Marc and Messier $^{38}$.]

1) The losses per unit area increase linearly with increasing film thickness, thus suggesting the movement of dipoles linked to the organic molecules. This is in accordance with the expression showing the relation of $P_{s}$ and $N$ (Eq. 4.9).

2) The losses due to the oxide layer are much higher than those due to the organic films, and particularly those due to a monolayer.

3) The $\tan \delta_{m}$ curve does not change much with frequency. The rise in the measurement frequency merely causes a slight displacement of the $\tan \delta_{m}$ curve towards higher temperatures.

4) The dielectric losses are much greater when the structure of the monolayer is disorganized (see Figure 9). However, the order of magnitude of tan $\delta_{m}$ is $10^{-3}$ for a compact monolayer which is smaller than expected. To explain this, these workers suggest that the $\mathrm{Ca}^{++}$ions considerably reduce the mobility of the polar groups by linking the molecules, and the rotation of a dipole around the molecular axis introduces fewer losses.

5) The two absorption peaks observed at $-100^{\circ} \mathrm{C}$ and $-30^{\circ} \mathrm{C}$ have been related to the degree of organization of the monolayers. Finally, based on similar peaks in several other types of organic substances, (in polyethylene a peak similar to that at $-100^{\circ} \mathrm{C}$; in stearon and nonapentacontanone a peak similar to that at $-30^{\circ} \mathrm{C}$ ) these peaks have been interpreted in terms of dipole movements. The respective peaks are thus thought to be due to the 
movement of the dipoles in the amorphous region of the monolayers, and due to the molecular rotation around the long axes in the crystalline parts of the layers.

The interpretation in terms of the movement of dipoles in these layers is not convincing because the measurements are carried out under homogeneous applied fields. In fact, motion of dipoles is possible only when non-homogeneous fields are applied. Since the soaps or metal salts of long chain fatty acids are known to be ionic substances forming stable monolayers (cf. Ref. 7, p. 193) there may be some ion content present in the deposited monolayers. Therefore, it appears to the author that the observed peaks may be attributed to the motion of these ions rather than the dipoles. The existence of amorphous regions in such organic monolayers, which these workers have suggested, has not yet been verified in any other experimental work. Nevertheless, this is the only systematic work on loss angle measurements which distinguishes between the various kinds of losses, and which has thus led to an estimate of the loss angle due to the Langmuir monolayers themselves. In presenting their measurements, MM were able to exclude the dielectric losses due to the natural oxide layer whose capacity was determined to be $920 \mathrm{nF} / \mathrm{cm}^{2}$.

It is interesting to point out the recent studies on $\tan \delta$ by $\mathrm{Nathoo}^{19}$ for a 23 layer film of stearic acid at room temperature over the frequency region $10^{-4}$ to $100 \mathrm{~Hz}$. Under these experimental conditions, no peaks were observed and the dielectric losses were found to decrease by an order of magnitude with increasing frequency in the range studied.

\section{ELECTRICAL CONDUCTION PROPERTIES}

Great emphasis was laid on studying electrical conduction through thin dielectric films, particularly in the period following the second World War. It was realized that the conductive properties of films should play an important role in the development of devices. Most of the earlier work on conduction behaviour has been done, however, using either vacuum deposited films or thermally grown oxide films on base metal electrodes. Electrical conduction in films has already been widely treated in the literature in recent years. The readers are advised to refer to the recent book by O'Dwyer ${ }^{69}$ who has given a comprehensive picture of the subject.

Because of the defect nature of the films, it is not possible to provide interpretation of experimental data using only one of the earlier concepts, such as field emission from a free-electron metal by Fowler and Nordheim ${ }^{70}$, metal-vacuum-metal junctions by Frenkel $^{71}$, or metal-insulator-metal junctions by Sommerfeld and Bethe ${ }^{72}$, etc. In fact, the conduction process has been recognized to be an involved process, and many co-operative phenomena such as space charge modulation (due to trapped carriers) or, say, the Poole-Frenkel effect or Schottky effect have been found to occur. In the context of this paper, basic theories of conduction phenomena in thin insulating films are discussed briefly just to remind the reader of the underlying basic concept. The simplest expressions used for the interpretation of experimental data are also given.

\subsection{Conduction Theories in Thin Films}

All the theories discussed here will apply to metalinsulator-metal systems, and the insulator in question may contain a significant proportion of impurities. In general the electron transport at a metal-insulator interface depends upon the nature of the contacts, and the conduction processes, in turn, are distinguished as follows:

1) If the contacts are ohmic $\left(\psi_{m}<\psi_{i}\right.$, where $\psi_{m}$ and $\psi_{i}$ are the work functions of the metal and insulator respectively) the conduction in the system is limited by the rate of electrons flowing through the bulk of the insulator, rather than the rate at which the electrons are supplied by the electrode. The process is then termed "bulk limited".

2) In the case of blocking contacts $\left(\psi_{m}>\psi_{i}\right)$, the rate of electrons flowing through the system is limited by the rate of electrons flowing over the interfacial barrier and the process is referred to as "electrode limited".

3) If the contacts are neutral, i.e. $\psi_{m}=\psi_{i}$, there is no necessity of charge transfer between the electrode and insulator. This situation corresponds to a transition between an ohmic and blocking contact. Under special circumstances, neutral contacts also occur if $\psi_{m}<\psi_{i}$ and the trap level is positioned more than $1 \mathrm{eV}$ above the Fermi level. Traps in insulating films arise because of the grain boundary defects or because of the large stresses, particularly in vacuum deposited films. These traps are an "extrinsic" property of the amorphous insulator, and their concentration may be as high as $10^{19}$ $\mathrm{cm}^{-3}$.

However, if the system is subjected to sufficiently high voltages, transition from bulk limited to elec- 
trode limited processes, or vice versa, may occur depending upon the nature of contacts. We shall be concerned here with several distinct conduction mechanisms only, and the discussion of theories will be limited to three voltage ranges: low, intermediate and high. The process is electrode limited for low and intermediate voltages and is bulk limited in the higher voltage region. In practice, however, many more co-operative phenomena may occur, because no clear cut criteria may be assigned to distinguish these three voltage regions. For greater details, reference should be made to a recent monograph ${ }^{73}$ on the subject.

\subsubsection{Fowler-Nordheim type tunnelling This} process requires that the system be subjected to high voltage biases so as to lower the barrier thickness at the negatively biased Fermi level to less than $50 \AA$. The well-known expression of Fowler-Nordheim type of tunnelling ${ }^{70}$ is then given by

$$
J=\frac{3.38 \times 10^{10} F^{2}}{\phi} \exp \left(-\frac{0.69 \phi^{3 / 2}}{F}\right),
$$

where $J$ is the current density, $F$ is the applied field and $\phi$ is the mean potential barrier height above the Fermi level of the cathode. This expression is true only if the ratio of the insulator effective mass to the free-electron mass $\left(\mathrm{m}^{*}\right)$ is unity. Otherwise the exponent of equation would become

$$
\left(\frac{-0.69\left(m^{*} \phi\right)^{3 / 2}}{F}\right)
$$

This process is electrode limited.

\subsubsection{Richardson-Schottky effect The current} flow through the insulator in this process is governed by the rate at which the electrons are thermally excited over the interfacial potential barrier into the insulator conduction band. Thus, the current flowing in the system is determined by Richardson's thermionic equation

$$
J=A T^{2} \exp \left(-\frac{\phi_{0}}{k T}\right),
$$

where $A$ is the well-known Richardson constant $\left(\left(4 \pi m e k^{2}\right) / \hbar^{3} \cong 120\right.$ if $\mathrm{J}$ is expressed in $\left.A \mathrm{~cm}^{-2}\right)$ and $\phi_{0}$ is the interfacial barrier height. However, when the applied field interacts with the attendant electrode image potential, it lowers the barrier height from $\phi_{0}$ and permits more current to flow over the barrier with increasing applied voltage. This effect is known as the Schottky effect ${ }^{74}$, and the barrier lowering is given by $\Delta \phi_{s}=\beta_{S} F^{1 / 2}$. The current is then determined by the Richardson-Schottky equation

$$
J=A T^{2} \exp \left(-\frac{\phi_{0}}{k T}\right) \exp \left(\frac{\beta_{\mathrm{s}} F^{1 / 2}}{k T}\right),
$$

where $\beta_{\mathrm{s}}$ is a constant $\left(=\mathrm{e}^{3} /\left(4 \pi \epsilon^{*} \epsilon_{0}\right)^{1 / 2}\right)$, and $\epsilon^{*}$ is the high frequency dielectric constant of the material. For sufficiently high fields across the interface, tunnelling may be the dominant conduction mechanism, and it is then governed by a suitably modified form of Eq. (5.1) in which the image force has been included.

5.1.3 Space charge limited currents In both of the processes discussed above, space charge effects were not considered. However, traps exist in most of the film systems used, and thus it is necessary to account for their contribution. First, the situation will be discussed for a trap-free insulator. In the case of ohmic contacts, a negative space charge region exists on the surface of the insulator, and an equal and opposite (positive) surface charge on the electrodes. When the field is applied, the space charge in the insulator increases and its redistribution takes place, depending upon the nature of voltage bias. After this redistribution, if the positive charge exists on the cathode, i.e. the cathode region acts as a charge reservoir to supply the required charge to the anode, the process is called "space charge limited" (SCL). For trap-free insulators, the expression derived by Mott and Gurney ${ }^{75}$ to calculate the current density is given by

$$
J=\left(\frac{9 \mu \epsilon \epsilon_{0}}{8 d^{3}}\right) V^{2},
$$

where $d$ is the insulator thickness. However, in practice, much lower currents have been observed, and they are found to be temperature sensitive, which could not be explained with the above equation. Rose $^{76}$ then pointed out that when the insulator contains traps, a large fraction of the injected space charge condenses into the traps. Thus, it was suggested to multiply the right-hand side of Eq. (5.4) by a quantity $\theta=\left(N_{\mathrm{c}} / N_{t}\right) \exp \left(-E_{t} / k T\right)$, where $N_{t}$ is the trap density., $E_{t}$ is the depth of the trap below the bottom of the conduction band, and $N_{c}$ is the effective density of states in the conduction band. Thus Eq. (5.4) may be expressed as:

$$
J=\left(\frac{9 \mu \epsilon \epsilon_{0} \theta}{8 d^{3}}\right) V^{2}
$$


Since $\theta$ is independent of $V$, the proportionality $J \propto V^{2}$, as in the case of trap-free insulator, still holds.

5.1.4 Poole-Frenkel effect When the applied field is high, the coulombic potential barrier of a donor centre or trap is lowered by an amount ${ }^{77}$

$$
\Delta \phi_{\mathrm{PF}}=\beta_{\mathrm{PF}} \mathrm{F}^{1 / 2}
$$

which is twice the attenuation of the barrier height due to the attendant image forces in the case of the Schottky effect. This occurs because the potential energy of an electron in a coulombic field is four times that due to the image force effects. $\beta_{\mathrm{PF}}$ is thus

$$
\beta_{\mathrm{PF}}=\left(\frac{e^{3}}{\pi \epsilon^{*} \epsilon_{0}}\right)^{1 / 2}
$$

This, in turn, amounts to the lowering of the ionization potential, Eg, of the donors in a solid. The bulk current density is then determined by the well-known Poole-Frenkel equation

$$
J=J_{0} \exp \left(\frac{\beta_{\mathrm{PF}} F^{1 / 2}}{2 k T}\right)
$$

where $\mathbf{J}_{0}=\mathrm{e} \mu \mathrm{N}_{\mathrm{c}} \mathrm{F}$ exp $(-\mathrm{Eg} / 2 \mathrm{kT})$ and is the. low field current density. Traps having a coulombic type of barrier would experience the PF effect at high fields, which results in the increase of the escape probability of immobile electrons therein ${ }^{78}$. The expression for insulators with shallow traps is given by:

$$
J=J_{0} \exp \left(\frac{\beta_{\mathrm{PF}} F^{1 / 2}}{k T}\right)
$$

The reader is reminded that the coulombic barrier exists not only between a donor centre and its electron but also between a charged trap and an electron. A charged trap is a trap which is charged when empty and neutral when full. Furthermore, Simmons ${ }^{73}$ has discussed another situation where the insulator contained both donor centres (lying below the Fermi level) and shallow neutral traps. The expression for the bulk I-V characteristic of the film is then given by

$$
J=J_{0} * \exp \left(\frac{\beta_{\mathrm{PF}} F^{1 / 2}}{2 k T}\right)
$$

where

$$
J_{0} *=e \mu N_{c}\left(\frac{N_{d}}{N_{t s}}\right)^{1 / 2} F \exp \left(-\frac{\left(E_{d}+E_{\mathrm{ts}}\right)}{2 k T}\right)
$$

The suffixes $d$ and $t s$ here refer to the donor and shallow traps, respectively. This equation holds even if there exists a range of trap and donor levels, and one of the trap levels is more dominant than the others.

\subsection{Conduction Mechanisms in Langmuir Films}

Of all the conduction mechanisms that are discussed above, the dominant one in Langmuir films seems to be tunnelling. Obviously, for measuring tunnelling currents, one needs to separate two metal electrodes by a barrier of $20 \AA-100 \AA$ in thickness which must be uniform, homogeneous and an excellent insulator. Thin oxide films grown on base metal electrodes were employed for such studies by Giaver ${ }^{79}$ and Nicol et $a l .,{ }^{80}$ because of their compactness and uniformity. The fact that Langmuir films of several organic materials may fulfill all these requirements for studying tunnelling was recognized by Miles and McMahon. ${ }^{81}$. These workers employed a monolayer of barium stearate $(\cong 25 \AA)$ as an insulating barrier between tin-lead and tin-indium evaporated film pairs. The experiments, however, did not prove useful, because an irreproducible and strongly timedependent resistance of the device was observed in these studies. This is not surprising, because the monolayer transfer technique was in its infancy and only a handful of electrical measurements had been performed prior to this work. It was also not known whether voids, defects or inhomogeneities in these films could be reduced to permit the construction of tunnel sandwiches. However, the studies of Miles and $\mathrm{McMahon}{ }^{81}$ provoked the interest of many others in the conduction behaviour of Langmuir films.

Handy and Scala ${ }^{4}$ subsequently tried to obtain new information on the conduction behaviour of Langmuir multilayers and considerable efforts were made by them to deposit compact films for their studies. In their extensive studies on $\mathrm{Ba}$ or $\mathrm{Ca}$ stearate films (1-10 monolayers) with counter-evaporated electrodes of $\mathrm{Sn}, \mathrm{Cu}, \mathrm{Ag}$ and $\mathrm{Pb}$ (and also using $\mathrm{Hg}$ as the counter-electrode in a few cases), the conduction characteristics were found to be highly non-linear and strongly temperature dependent. Figure 10 shows the typical behaviour of a symmetric Sn-3 layer calcium

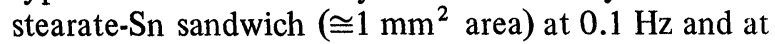




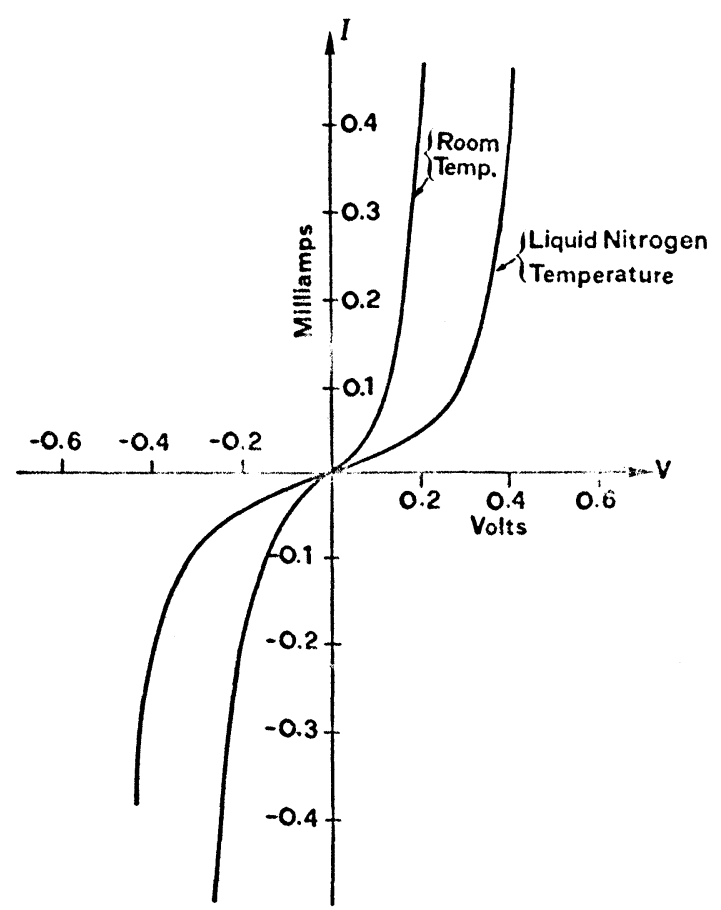

FIGURE 10 Current-voltage characteristics at $0.1 \mathrm{~Hz}$ of Sn -3 layer calcium stearate-Sn sandwich of $\sim 1 \mathrm{~mm}^{2}$ area at room and liquid nitrogen temperatures. Polarity is that of organic coated tin base layer. [After Handy and Scala ${ }^{42}$.]

room and liquid nitrogen temperatures. HS further studied the behaviour of a 3-layer and a 5-layer thick film at constant voltages for two junctions as shown in Figure 11. Detailed analysis of the temperature dependence of conduction for a 3-layer sandwich led them to believe that the electron tunnelling is the conduction mechanism at low temperatures $\left(<-100^{\circ} \mathrm{C}\right)$ and small thickness, and thermionic emission at high temperatures $\left(>40^{\circ} \mathrm{C}\right)$ and larger thicknesses. A superposition of both of these mechanisms has, however, been assumed by them at an intermediate temperature range. Handy and Scala also determined the zero voltage thermal activation energy $\phi_{b}$ as $0.25<\phi_{b}<0.30 \mathrm{eV}$ by plotting the thermionic contribution to the current on a Schottky plot of $\log \left(\left(I-I^{\prime}\right) / T^{2}\right)$ vs. $1 / \mathrm{T}$ as shown in Figure 12. In an attempt to analyse their results using theoretical expression derived by Stratton $^{82}$ and Simmons, ${ }^{83}$ they failed to obtain a satisfactory fit of the observed data. Consequently no satisfactory explanation could be given for the observed temperature dependence. In fact, they have shown that the fatty acid films are

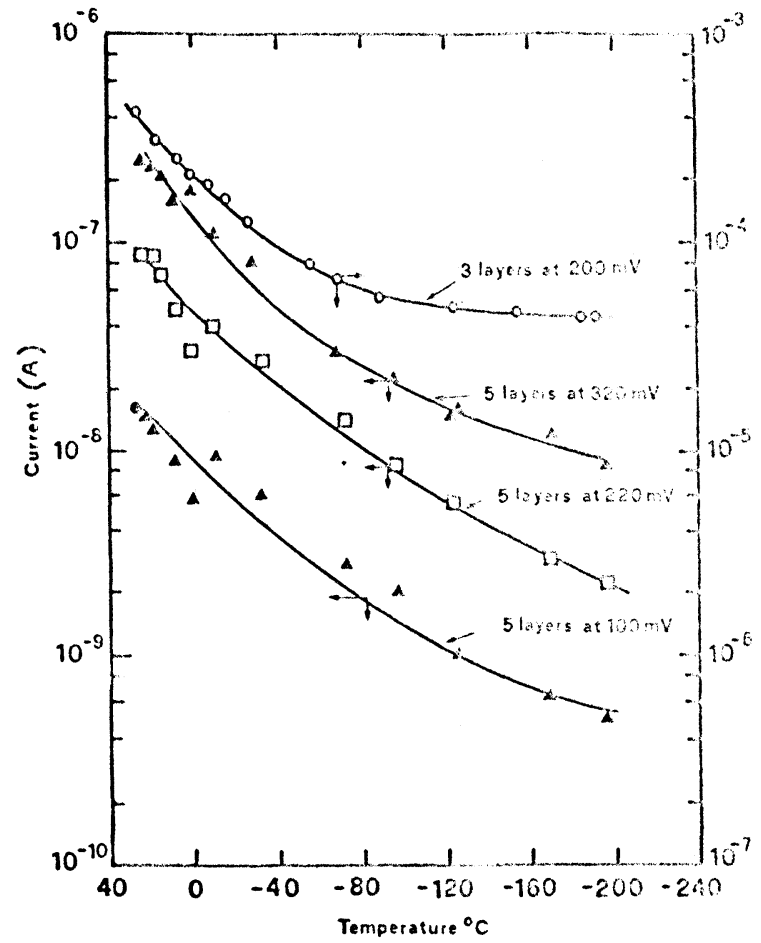

FIGURE 11 Plot of current vs. temperature at constant voltage for $\sim 3$ layer thick film (uppermost curve) and for $\sim 5$ layer thick film (three lower curves). Polarity of test voltage is organic coated base electrode positive. [After Handy and Scala $^{42} .1$

promising for electron tunnelling, and the low values of $\phi_{b}$ could be due to the defects and impurities within the film. One of the important conclusions is the necessity of a reactive and hence oxidized surface for the formation and retention of low porosity layers. If noble metals (e.g. $\mathrm{Ag}$ or $\mathrm{Au}$ ) were used as the first electrode, the junction resistance was found to be less than $1 \Omega$, and none of the gold-based samples were successful for electrical studies.

Horiuchi et al. ${ }^{84}$ employed calcium stearate films sandwiched between asymmetric electrodes of tin and aluminium to study the voltage and temperature dependence of the current. As is shown in Figure 13a the observed current $I_{1}$ was found to be strongly temperature dependent above $200^{\circ} \mathrm{K}$ and nearly independent below this temperature. This was the situation when the lower tin electrode was negatively biased. Conversely, when the upper aluminium electrode was negatively biased, the current $I_{2}$ was observed to be temperature independent in the temperature range studied. This type of behaviour of MIM junctions suggested that more than one mechan- 


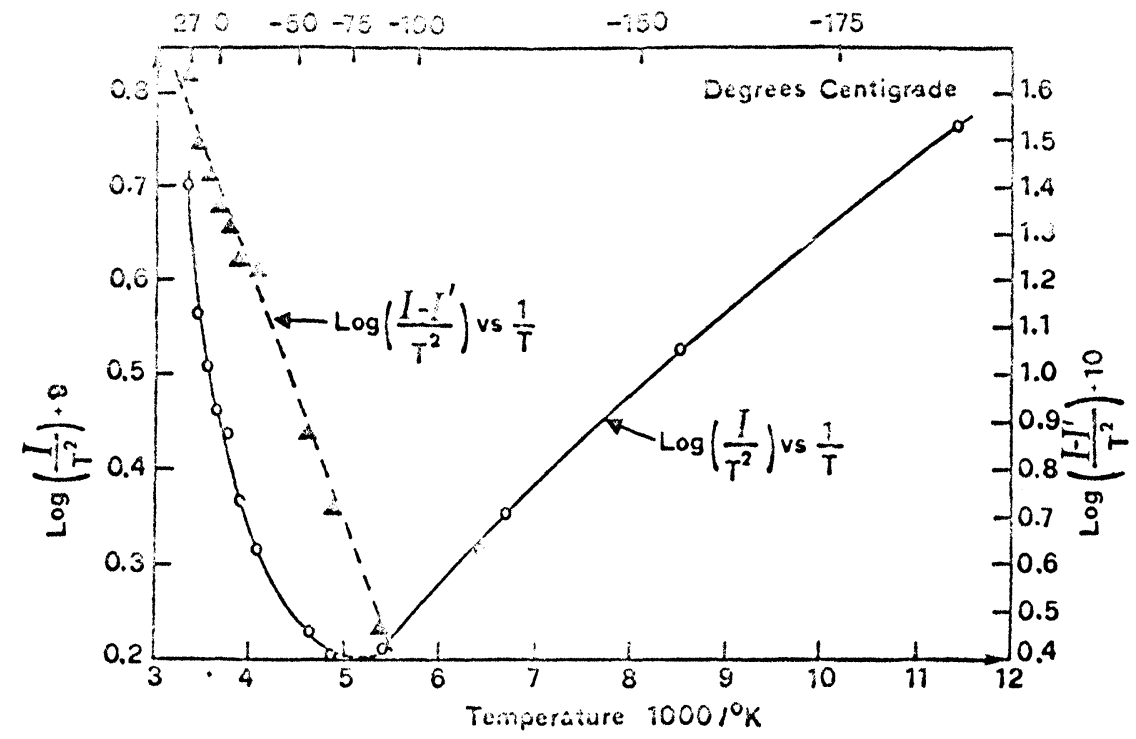

FIGURE 12 Plot of $\log \left(I / T^{2}\right)$ vs. $(1 / T)$ and $\log \left(\left(I-I^{\prime}\right) / T^{2}\right)$ vs. $(1 / T)$ for 3 layer sandwich, where $I=$ total current, $I^{\prime}=$ low temperature current in amperes and $T=$ absolute temperature. [After Handy and Scala ${ }^{42}$.]

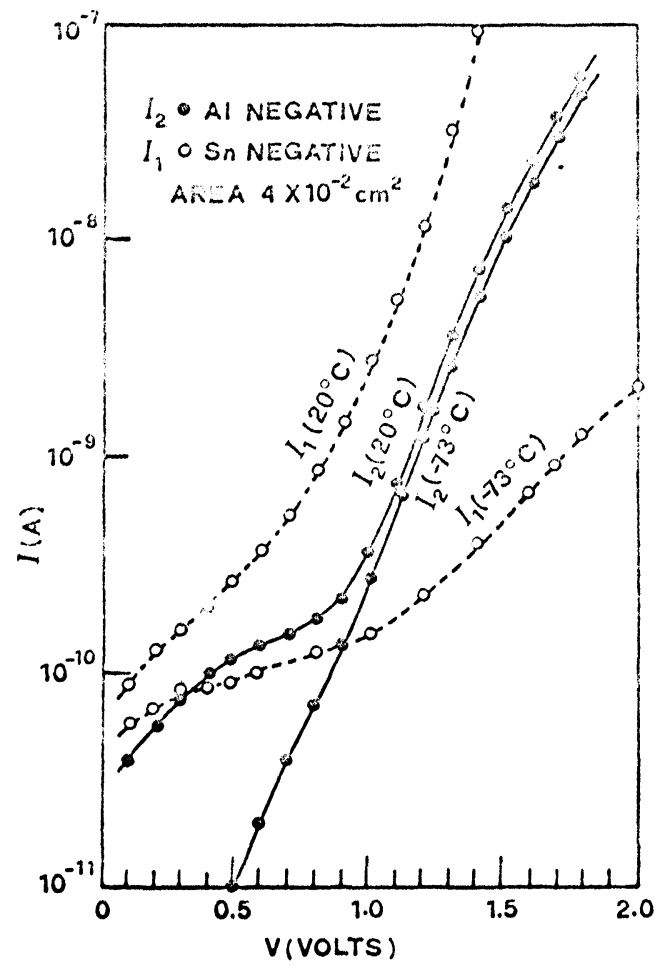

(a)

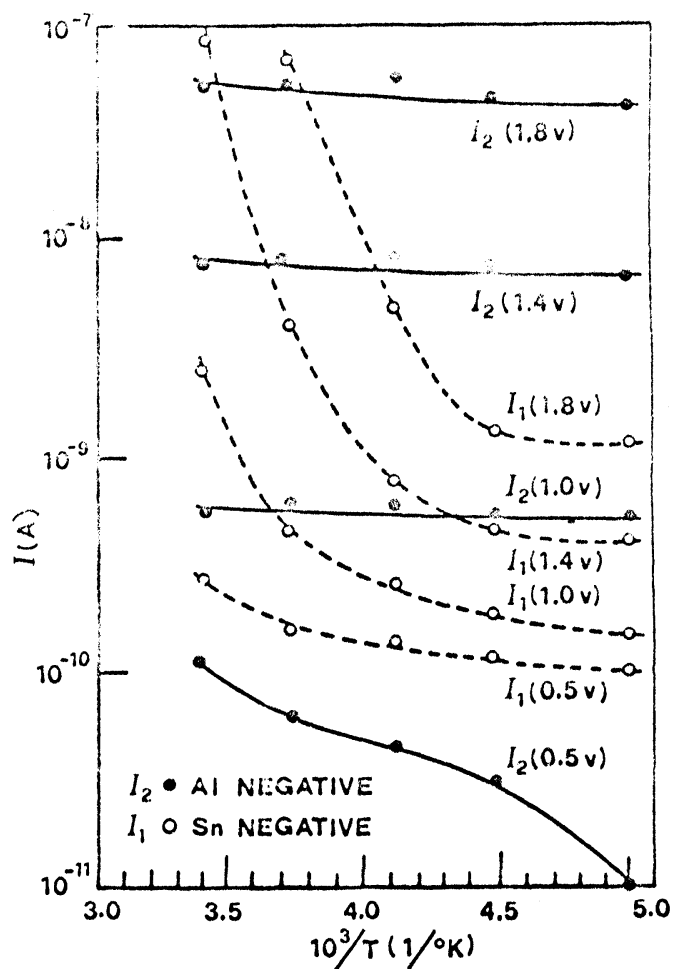

(b)

FIGURE 13 (a) Plot of $I_{1}$ and $I_{2}$ vs. V at fixed temperature for $\mathrm{Sn}-3$ layer Ca stearate-A1 junctions; and (b) plot of $I_{1}$ and $I_{2}$ vs. $1 /$ T at fixed voltage for Sn-Ca stearate junctions. Black dots and white circles represent $I_{2}$ and $I_{1}$, respectively. [After Horiuchi et $a l^{84}$.] 
ism of electron transfer is operative (see Figure 13b). Horiuchi $e t$ al. analysed their V-I characteristics using the trapezoidal energy barrier model of Simmons 83,85 and Pollack et al. ${ }^{86,87}$ and thus determined barrier heights $\phi_{b}$ at the two interfaces; $\phi_{b}=1.3 \mathrm{eV}$ (for stearate-aluminium) and $\phi_{b}=0.8 \mathrm{eV}$ (stearate-tin). This indicated that the work function of tin is lower by $0.5 \mathrm{eV}$ than that of aluminium, which is in contradiction to the known difference of $1.5 \mathrm{eV}$. This discrepancy, however, could not be resolved by these workers even when they studied symmetric junctions of tin electrodes and took the natural oxide layer into consideration.

From their detailed analysis it was shown that the temperature and voltage dependence of the current satisfies the Schottky emission mechanism, whereas tunnelling has been predicted from the voltage dependence of the low temperature current. Alternatively, the two mechanisms have been found to be operative for the negatively biased lower Sn electrode and the upper A1 electrode, respectively. To resolve the difference in the barrier height determined from

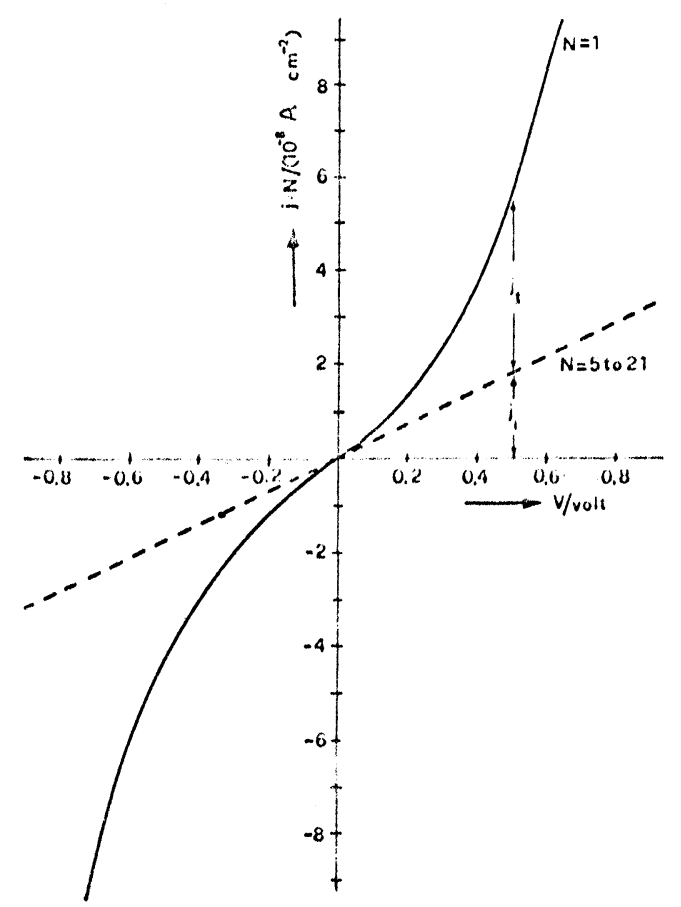

FIGURE $14 \mathrm{jN}$ vs. $\mathrm{V}$ for A1-Cd stearate-Hg devices when A1 electrode positively biased. The dashed curve $\mathrm{N}=5$ to 21 ; $\mathrm{j}=\mathrm{j}_{\mathrm{i}}$ (impurity dependent current), proportional to $\mathrm{V}$ and $1 / N$. Full curve $N=1 ; j=j_{t}+j_{i}\left(j_{t}\right.$ tunnelling current $) . j_{t}$ surplus over $j_{i}$ is given by dashed curve. [After Mann and $\mathrm{Kuhn}^{15}$.] the two operative mechanisms, they have assumed the presence of some conductive patches in the films which would let the tunnel current pass through them readily. This seems reasonable, as tunnelling emission was found to be more strongly dependent on the barrier height than the Schottky emission. From their studies of the $I-V$ characteristics, which were symmetrical with respect to the polarity of applied voltage, the dominant conduction mechanism has been suggested to be barrier limited because the characteristic was dependent on electrode material. To avoid the formation of oxide layers, efforts were made to study gold based devices, but measurements could not be carried out because the samples prepared showed ohmic characteristics with very small resistance.

Unlike the measurements of previous work$\operatorname{ers}^{42,81,84}$, Mann and co-workers ${ }^{15,88}$ succeeded in giving a quantitative proof of the tunnelling theory in their measurements of dc conductance through Langmuir films of a few fatty acid salts. The success of their measurements lies in the fact that film deposited by using a modified B-L technique ${ }^{23}$ did not possess any holes and conducting imperfections. Analysis of the experimental data was made in accordance with the concept of quantum-mechanical tunnelling proposed by Sommerfeld and Bethe. ${ }^{72}$

To determine the conductivity, Bethe's relation ${ }^{72}$ for small values of applied voltages'was used, which is expressed as:

$$
\sigma_{t}=\left(\frac{e^{2}}{\hbar^{2}}\right)(2 m \phi)^{1 / 2} \exp \left[-\left(\frac{2 d}{\hbar}\right)(2 m \phi)^{1 / 2}\right]
$$

where $\phi$ is the difference between the work function of the metal and the electron affinity of the insulator. All other symbols have their usual significance. This equation refers to equal metals and, if the metal electrodes are different, $\phi$ should be replaced by

$$
\phi=\left(\frac{2}{3} \frac{\phi_{1}^{3 / 2}-\phi_{2}^{3 / 2}}{\phi_{1}-\phi_{2}}\right)^{2}
$$

where $\phi_{1}$ and $\phi_{2}$ are the $\phi$ values for the respective metal electrodes. Since each insulator is expected to have some impurity-dependent conductivity $\sigma_{i}$ independent of $d$, besides the tunnelling conductivity $\sigma_{t}$, Mann et al have analysed the results by separating out these two contributions from the experimental total conductivity $\sigma\left(=\sigma_{i}+\sigma_{t}\right)$. 
Mann and Kuhn ${ }^{15}$ investigated many samples in the thickness range containing 5 to 21 layers with a lower $\mathrm{Al}$ electrode biased both positively and negatively. The measurements of dc conductance at $20^{\circ} \mathrm{C}$ and $-35^{\circ} \mathrm{C}$, with various upper electrode materials, indicated that the logarithm of the current density $j$ is proportional to $V$ and inversely proportional to $d$, as is expected. Figure 14 is a typical plot of $N j$ versus $V$ of cadmium arachidate film, where $N$ refers to the number of layers. The dotted line represents the current due to impurities $j_{i}$ and the other curve for $\mathrm{N}=1$ gives the total current $j$ which is a sum of $j_{i}$ and $j_{t}$ (tunnelling current). In Figure 15,

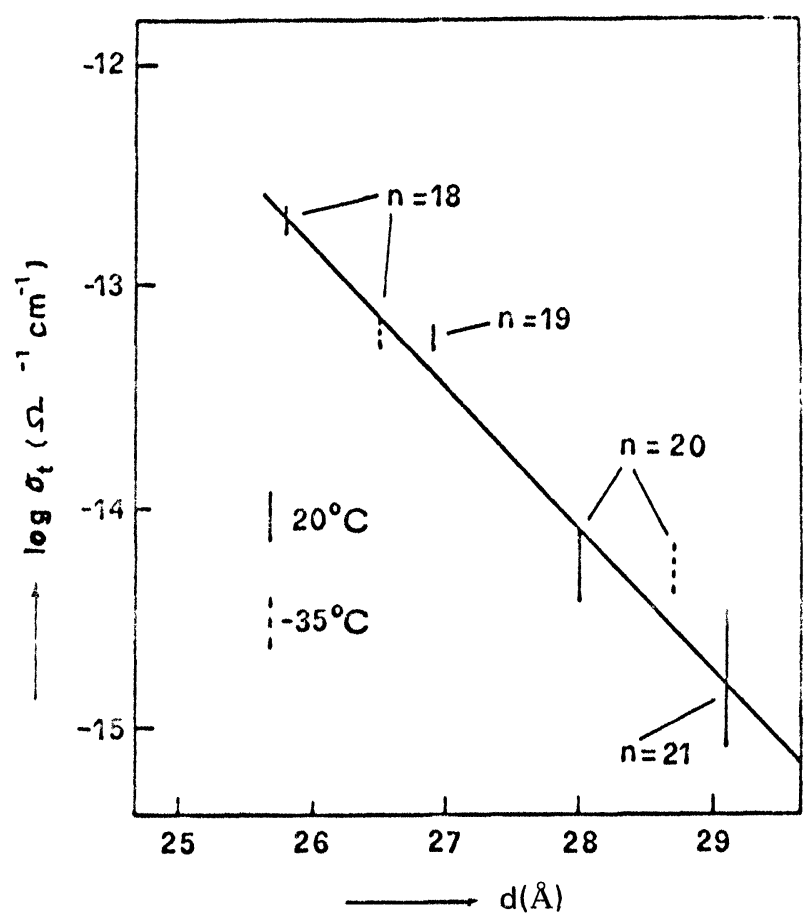

FIGURE 15 Logarithm of $\quad \sigma_{\mathrm{t}}=\left(\mathrm{j}_{\mathrm{t}} \mathrm{d} / \mathrm{V}\right) \mathrm{V} \rightarrow 0 \quad\left(\sigma_{\mathrm{t}}\right.$ tunnelling conductivity) vs. $d$ for $A 1-\mathrm{Cd}$ salt of $\mathrm{CH}_{3}$ $\left(\mathrm{CH}_{2}\right)_{\mathrm{n}-2} \mathrm{COOH}-\mathrm{Hg}$ devices at $20^{\circ} \mathrm{C}$ and $-35^{\circ} \mathrm{C}$ with $\mathrm{n}=18-21$. [After Mann and $\mathrm{Kuhn}^{1 \mathrm{~s}}$.]

$\log \sigma_{t}\left(\sigma_{t}=\sigma-\sigma_{i}\right)$ is plotted against thickness $\mathrm{d}$ for four types of cadmium salts of the fatty acids $\mathrm{CH}_{3}$ $\left(\mathrm{CH}_{2}\right)_{n-2} \mathrm{COOH}$ with $n=18-21$. Evidently, the experimental points for both 20 and $-35^{\circ} \mathrm{C}$ are on the same straight line in accordance with Bethe's equation. This exponential decrease of conductivity with increasing thickness of the insulating barrier $d$ verifies the tunnelling theory. The vacuum work functions of $\mathrm{Al}$ and $\mathrm{Hg}$ determined in their work theoretically were found to be in good agreement with the known values.

Further experiments were performed to measure the photocurrent in the sandwich assemblies between similar and dissimilar electrodes. From these measurements, the vacuum work functions of $\mathrm{Al}$ and $\mathrm{Hg}$ were determined independently, and the excellent agreement between theoretical and experimental values led to the belief that large imperfections were absent in the monolayer structures. Absence of even small numbers of holes and conducting imperfections, which strongly influence the resistance of the devices, have been verified quantitatively. These workers ${ }^{8.8}$ had also verified the tunnelling theory in a previous work on photographic sensitization. Fatty acid layers were used as "distance keepers" in the sensitizers to distinguish between an energy transfer mechanism and an electron injection mechanism. The dye was still found to be sensitized, which led to the conclusion that the sensitization is due to the energy transfer mechanism, since the electron tunnelling through fatty acid monolayers was extremely improbable. ${ }^{89}$

Strong evidence for tunnelling at low voltage has been presented by Leger et al. ${ }^{55}$, who studied electronic transport through organic films of cyanine with two grafted stearic chains $\left(\mathrm{St}_{2} \mathrm{Cy}\right)$. A typical $I-V$ characteristic at $1.5^{\circ} \mathrm{K}$ is shown in Figure 16 for a sample $\mathrm{A} 1$-cyanine- $\mathrm{Pb}$ for different voltage ranges. The curve (B) is quadratic for voltages less than $0.1 \mathrm{~V}$ and exponential at higher voltages. Such behaviour is clearly not compatible with a conductance dominated by pinholes in the organic film and tunnelling through the natural oxide layer. In another curve (A), the energy gap of superconducting lead $\left(\Delta_{\mathrm{Pb}}\right)$ is clearly visible. Another striking feature of the characteristic is its non-linear behaviour for voltages $>50 \mathrm{mV}$ which, together with the above observations, led to the conclusion that tunnelling is the dominant conduction mechanism at low voltages. However, contradictory results have been obtained in similar work on Langmuir films of stearic acid containing 5 layers $^{19}$. Figure 17 shows a plot of $\log I$ versus $V^{1 / 2}$ at room temperature which fits well an expression of the form $I \propto \exp \beta V^{1 / 2}$ with $\beta=1.8 \times 10^{5}$ $(V m)^{1 / 2}$. Predicted theoretical values of $\beta$ are $\beta_{\mathrm{PF}}=4.7 \times 10^{5}(\mathrm{Vm})^{1 / 2}$ for Poole-Frenkel conduction and $\beta_{S}=2.3 \times 10^{5}(\mathrm{Vm})^{1 / 2}$ for Schottky conduction. From such a comparison it was concluded that the latter is the dominant mechanism in stearic acid films. Dominance of Schottky conduction mechanism in Langmuir films in the range of 1-10 


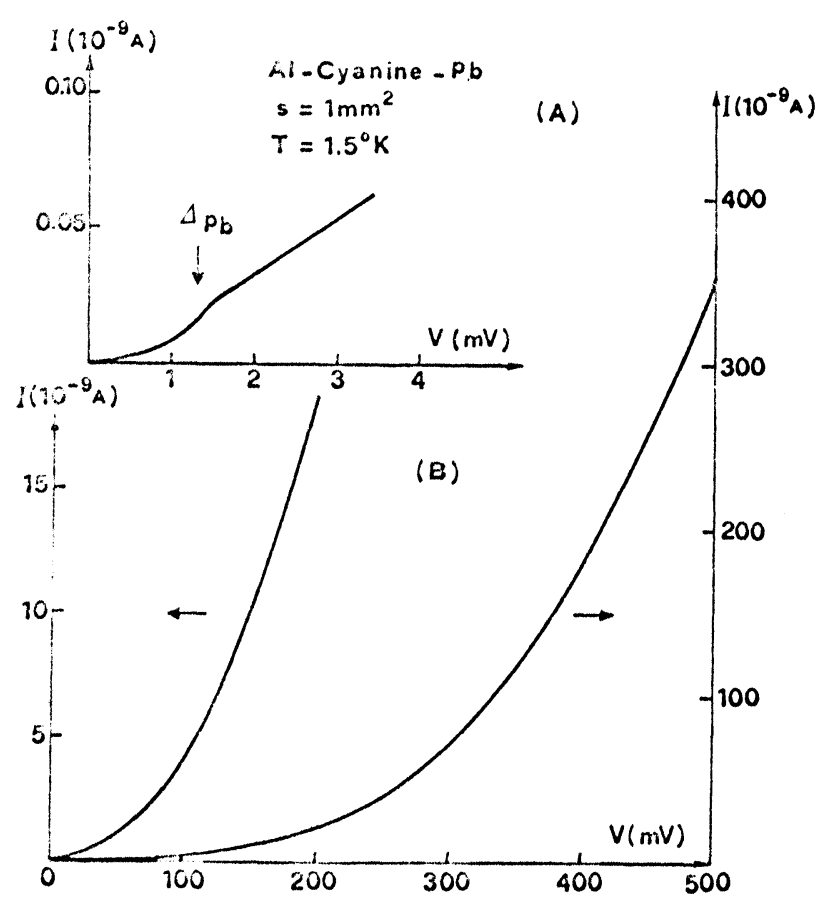

FIGURE 16 Plot of current vs. voltage for A1-cyanine-Pb device of area $\sim 1 \mathrm{~mm}^{2}$ at a temperature of $1.5^{\circ} \mathrm{K}$ (curve $\mathrm{A}$ ). Both the curves (A) and (B) are plotted to show the behaviour in different voltage ranges. The appearance of the superconducting lead gap $\Delta_{\mathrm{Pb}}$ is marked in the curve (A).

monolayers has also been proposed by Srivastava, ${ }^{90}$ based on experimental studies of the thickness dependence of the breakdown field (described later in detail).

All the conduction studies, described above, have been carried out in the low thickness range of Langmuir films (1-10 monolayers) and different conduction processes have been found operative under different conditions. However, careful examination of existing data shows that the tunnelling mechanism is dominant even though there are some indications of Schottky conduction. In fact, tunnelling has been found to be the dominant conduction mechanism in many other types of film systems in the comparable thickness range. On the other hand, it has been indicated ${ }^{73}$ that Schottky barriers in thin films are much more easily investigated using the ac technique. Nevertheless, to make sure that tunnelling is the operative conduction mechanism in Langmuir films, more experiments are required.

Since tunnelling currents are known to have only a slight temperature dependence, and the Schottky currents depend strongly on temperature, such

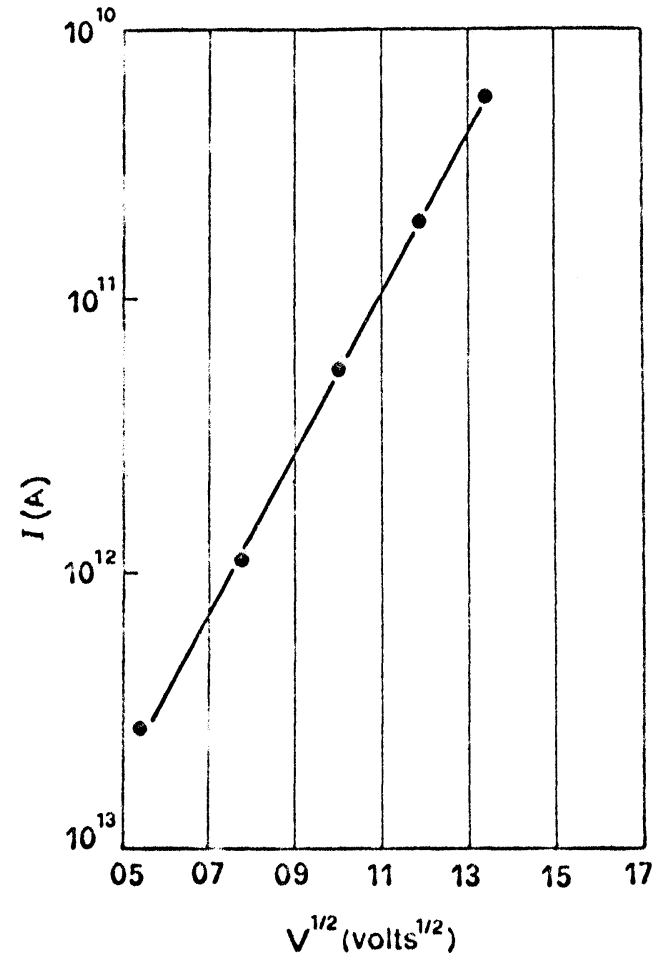

FIGURE 17 Current-voltage characteristic for a 5 layer thick stearic acid film at room temperature plotted as $\log$ I vs. $\mathrm{V}^{1 / 2}$. [After Nathoo ${ }^{19}$.]

temperature-dependent studies may prove useful for the choice of the conduction mechanism in these films. Unfortunately, most of the data have been interpreted without considering the nature of contacts, which have a lot of influence on the underlying conduction process. It is essential to examine the nature of contacts in future studies on these films. The presence of a space charge region in MIM structures has also not been accounted for in any of the measurements up to now, and this is perhaps the reason that some workers have indicated more than one conduction process to be operative, but failed to determine what exactly it is. Due to the lack of any judicious study which accounts well for all the factors influencing the conduction process, and in the light of the existing contradictory experimental evidence, it is premature to conclude anything about the conduction process in Langmuir films.

\subsection{High Field Conduction Characteristics}

Multimolecular films of stearic acid were chosen for high field conduction studies because of their ordered 
structure and because such films had been used by many workers in the study of tunnelling phenomena. Nathoo and Jonscher ${ }^{91}$ studied the behaviour of these films under high applied (ac and dc) fields to get more information about their dielectric properties. A sandwich structure of the type A1-film-A1 was used, and the measurements were made by placing the device in vacuum, since exposure to air caused reversible increases of the dc conductivity by an order of magnitude. A thickness range of 6-40 monolayers was chosen.

The response of the metal-film device to a step voltage was characterised by an initial surge followed by a gradual decay which lasted for hours, and sometimes for several days in thicker films. At room temperature the current was time dependent following the relation $I \propto t^{\mathbf{0 . 7}}$. On removal of a steady bias and shorting of the sample, a corresponding discharge current was observed. Figure 18 shows a typical
$I \propto E^{\prime .25}$. At fields in excess of $10^{5} \mathrm{~V} \mathrm{~cm}^{-1}$ the characteristic, however, was found to obey Poole's $\operatorname{law}^{92}$ of the form

$$
I \propto \exp \left(\frac{e S E}{k T}\right)
$$

where $2 \mathrm{~S}$ is the distance between donor-like Poole centres, ${ }^{93}$ i.e. neutral when occupied by the charge in question. To determine the value of $2 S$, Nathoo and Jonscher obtained the $I-V$ characteristics for a film containing 11 layers at four different temperatures and found that each of the characteristics obeys Poole's law. At temperatures between $198^{\circ} \mathrm{K}-295^{\circ} \mathrm{K}$, the values of $2 S$ varied between $33 \AA$ and $40 \AA$. It is expected that the donor-like centres should be separated by an integral multiple of the molecular length of the fatty acid film under investigation. In the present case, they ${ }^{91}$ expected that the donor-like

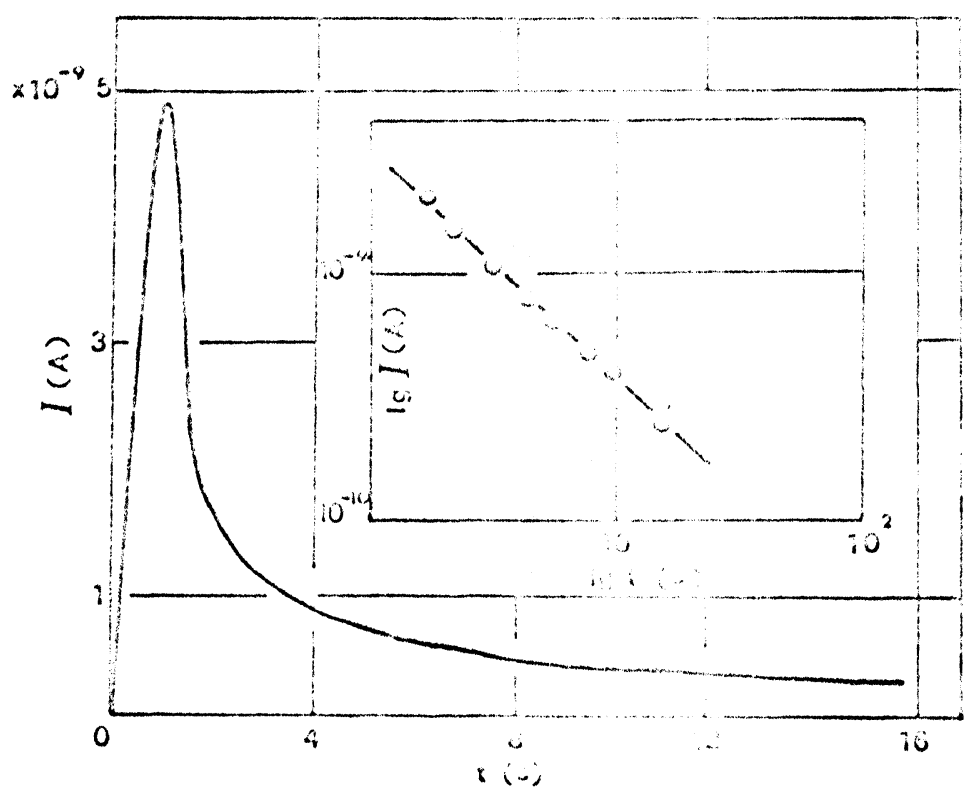

FIGURE 18 Recorder tracing of the current I after switching on a steady field for A1-8 layers thick stearic acid film-A1. The insert is a corresponding $\log \mathrm{I}-\log \mathrm{t}$ plot giving a slope of -0.7 . [After Nathoo and Jonscher ${ }^{9}{ }^{1}$.]

recorder tracing of the decay current after switching on a dc field for an 8 layer thick film, which follows the $I \propto t^{n}$ behaviour with $t=-0.7$. The $\mathrm{dc}$ characteristic shown here was obtained by subtracting the discharge current from the charging current. Further, the current was slightly non-linear at fields $<10^{5} \mathrm{~V} \mathrm{~cm}^{-1}, \quad$ varying according to centres are probably located at th? more reactive $\mathrm{COOH}$ end group of the molecules which separate them by twice the molecular length $(\cong 50 \AA)$. Since the observed values of $2 S$ were found to lie between one and two molecular lengths, the discrepancy can be explained by assuming an effective carrier temperature $T_{c}$ in excess of the ambient lattice temper. 
ature 94 . Subsequently, these authors measured "low field" ac conductance $\sigma(\omega)$ at peak amplitudes $<10^{5}$ $\mathrm{V} \mathrm{cm}{ }^{-1}$, and observed a relatively weak temperature dependence of the absolute magnitude of $o(\omega)$. Figure 19 is a plot of $\sigma(\omega)$ as a function of the

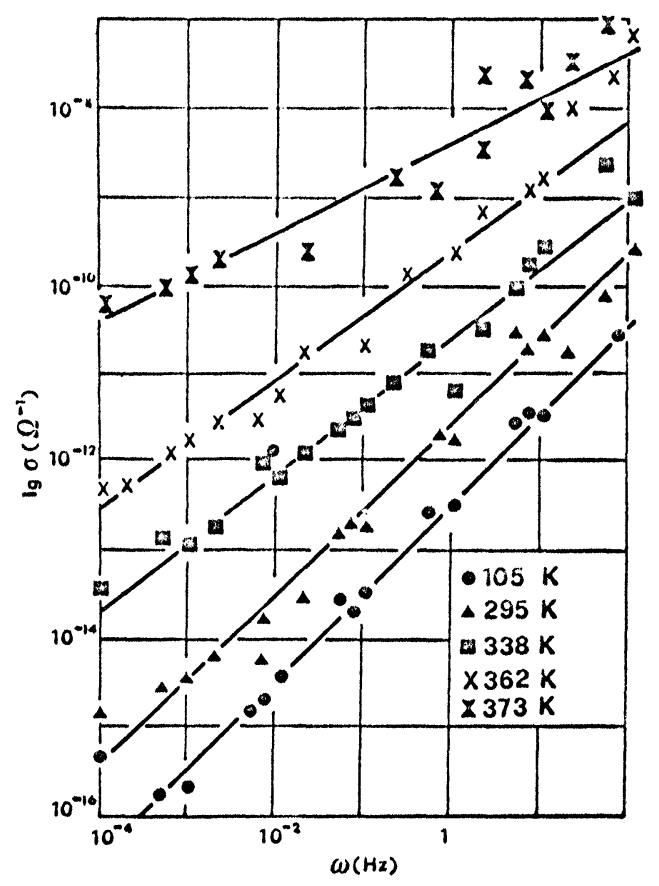

FIGURE 19 The ac conductivity $\sigma(\omega)$ vs. frequency $\omega$ for 11 layer thick film with the specimen area $\sim 1 \mathrm{~mm}^{2}$. The conductance scale refers to the lowest temperature, higher temperature characteristics being successively displaced by one decade for clarity. [After Nathoo and Jonscher ${ }^{9} 1$.]

frequency for a stearic acid film (11 layers thick) at different temperatures. Evidently, all the characteristics may be represented by the power law dependent on frequency of the form

$$
\sigma(\omega) \propto \omega^{n},
$$

where $n$ is temperature dependent and varies between $0.5<n<1$ at high and low temperatures, respectively. Similar behaviour of $\sigma(\omega)$ was observed in many other hopping systems, but these were mostly disordered or amorphous. From the fact that organic films, which are known to be largely ordered, have also shown a similar behaviour, they concluded that the frequency dependence of $\sigma$ is a general feature of hopping systems and not restricted to disordered solids. Regarding the nature of the hopping charges, their suggestion is that these could be electrons or protons, and much less likely heavier ions. Later, Jonscher ${ }^{95}$ has justified the observed behaviour of $\sigma$ in these ordered systems. He explained that even a perfectly ordered system in which all "hops" had equal probability distribution, the actual delay times would vary over a certain range, which could give rise to the observed dependence.

Further measurements were made by these workers 96 in the "high field" regime, extending upto $2 \times 10^{6} \mathrm{~V} \mathrm{~cm}^{-1}$ for a wide range of frequencies. In particular, their interest was to get information on whether or not the time- and frequency-dependence of the response was a function of the amplitude of the electric field. All the measurements were made on a 5-layer thick stearic acid film sandwiched between aluminium electrodes. Figure 20 shows the frequency domain measurements on two areas of the same film for a wide range of field amplitudes. Curve (a) shows that the results are different only in the lower

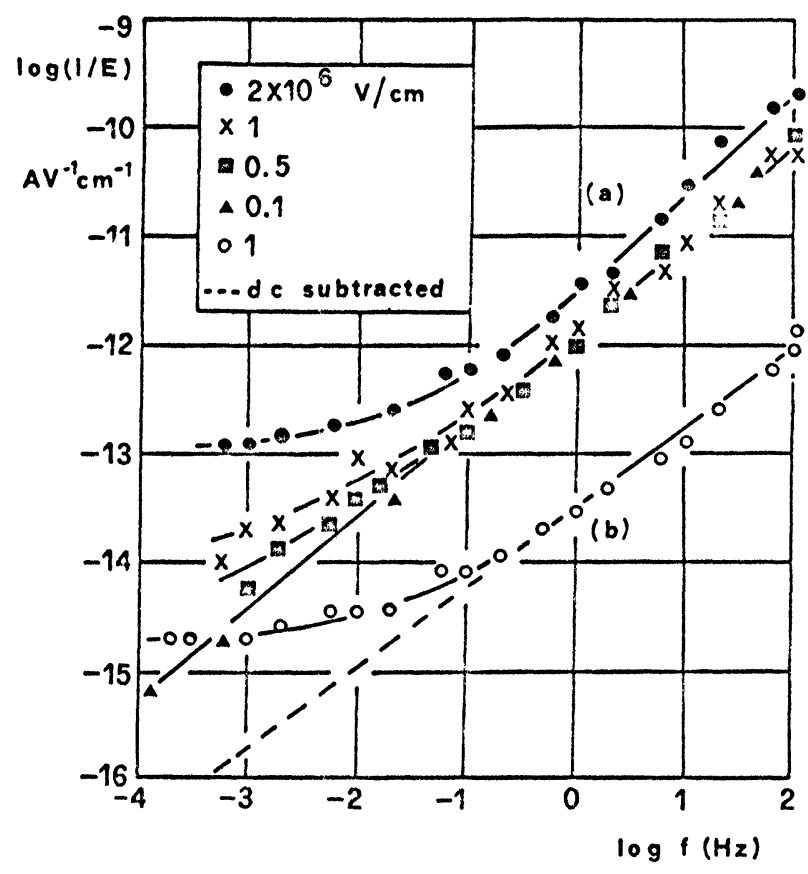

FIGURE 20 Frequency domain measurements on two areas (a) and (b) of the same device, A1-5 layer stearic acid film-A1, for a range of electric fields at room temperature. The dashed line for sample (b) represents the "true ac" data afer deduction of the dc. Data for sample (b) are displaced downwards by two orders of magnitude for clarity. The curves represent $\log \log$ plots of $(\mathrm{I} / \mathrm{E})$ vs. $\mathrm{f}$ where $\mathrm{I}$ is the current per $\mathrm{cm}^{2}$ and $\mathrm{E}$ is the field in $\mathrm{V} / \mathrm{cm}$. [After Jonscher and Nathoo ${ }^{96}$.] 
frequency region $\left(<0.1 \mathrm{H}_{\mathrm{z}}\right)$ which is due to the increasing level of $\mathrm{dc}$ conduction and is strongly non-linear for fields over $10^{5} \mathrm{~V} \mathrm{~cm}-1$. Curve (b) represents a similar characteristic taken at $1 \times 10^{6} \mathrm{~V}$ $\mathrm{cm}^{-1}$ when the dc level was subtracted from the total current. On extrapolation of the high frequency line, the exponent of Eq. (5.15) $n \cong 0.8$ was found. Obviously, the entire characteristic at a field of $2 \times 10^{6} \mathrm{~V} \mathrm{~cm}^{-1}$ shifts upwards by a factor of about 2.5 in the frequency region studied, but its slope does not change much. Thus it has been suggested that the onset of the frequency dependence of the characteristic does not change significantly because of the onset of ac non-linearity.

Time domain measurements were made on a different area of the same film, and the plots of charge currents (after subtracting the steady dc current) and discharge currents versus time are shown in Figure 21. The data give a dependence of the type $I \propto t^{-n}$ which, in the linear regime, gives the conductivity dependence of the type given by Eq. (5.15). The slope of discharge currents increases from 0.74 at the "low fields" $\left(2.25 \times 10^{5} \mathrm{~V} \mathrm{~cm}^{-1}\right)$ to 0.87 at the

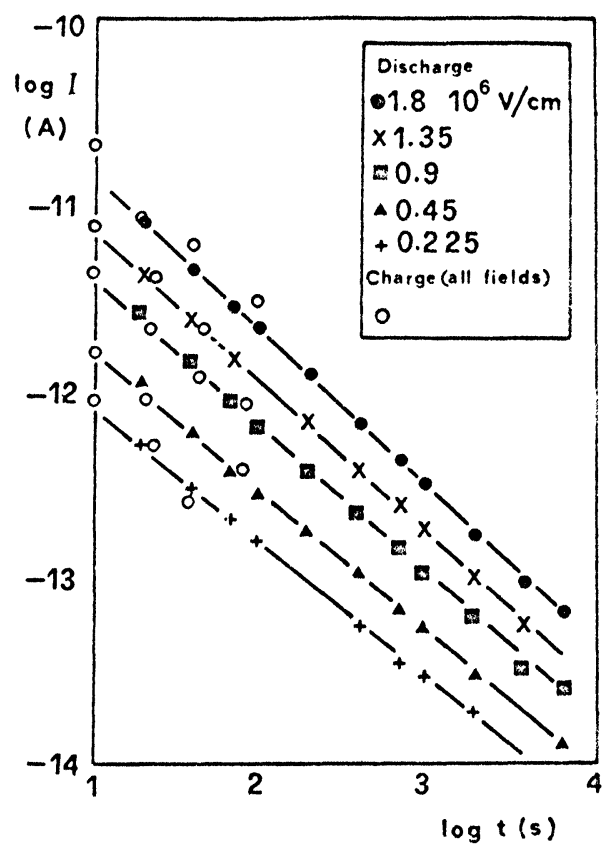

FIGURE 21 Time domain measurements of the discharge currents (symbols indicated) and charge currents (open circles) for a different area of the same film as in Figure 20, for different charging fields. Charge currents have dc subtracted. The curves are $\log -\log$ plots of I vs. $t$ (time). [After Jonscher and Nathoo ${ }^{96}$.] "high fields" $\left(1.8 \times 10^{6} \mathrm{~V} \mathrm{~cm}^{-1}\right)$. The discrepancy between the field-dependent slopes in the time domain and in the frequency domain is attributed more to the inapplicability of the Fourier transformation in the non-linear regime. Even the theoretical suggestion ${ }^{97}$ that the effect of a high field on the step function response be represented to a first approximation by a change of the amplitude of response without a change of time dependence, could not be strictly applicable in the present measurements. However, it has been used to explain the results as a reasonable first approximation, which then permits Fourier transformation in the non-linear regime. Validity of such an approximation is further supported by the fact that a small but definite increase of capacitance with increasing field has been observed in both the time and frequency domain measurements. Such behaviour has also been reported in measurements on silicon oxide ${ }^{98}$ and is also expected theoretically. ${ }^{97}$

The whole range of data has been considered as evidence for a small but definite effect of high fields on the time dependence of step function response which is more pronounced in the time domain than in the frequency domain. Such a definite trend could not be determined in the frequency domain measurements on silicon oxide. ${ }^{98}$ Although the results have been interpreted qualitatively by the authors ${ }^{91,96}$, no conclusion has been reached regarding the transient behaviour of such films under high applied fields (dc) or about their ac conductivity. Nevertheless, they have opened up a new series of measurements, and further measurements along these lines would yield many more facts about the high field conduction characteristics of Langmuir films. Most interesting would be to discover the nature of the hopping charges by studying the field assisted hopping mechanism between closely spaced donor-like centres in several other organic compounds.

\section{REFERENCES}

1. V. K. Agarwal, Thin Solid Films 24, 55 (1974).

2. Lord Rayleigh, Phil Mag., 48, 321 (1899).

3. N. K Adam, The Physics and Chemistry of Surfaces (3rd ed., Oxford University Press, London, 1941) Chap. 2.

4. I. Langmuir, Trans. Faraday Soc. 15, 62 (1920).

5. K. B Blodgett, J. Am. Chem. Soc. 57, 1007 (1935).

6. K. B. Blodgett, and I. Langmuir, Phys. Rev. 51, 964 (1937).

7. G. L. Gains, Jr., Insoluble Monolayers at Liquid-Gas Interfaces (Wiley, New York, 1966).

8. V. K. Srivastava, Physics of Thin Films (Academic Press, New York, 1974) 7, 311.

9. W. D. Harkins, Physical Chemistry of Surface Films (Van Nostrand-Reinhold, Princeton, 1954). 
10. A. W. Adamson, The Physical Chemistry of Surfaces, (Wiley, New York, 1960).

11. A. E. Alexander Rep. Prog. Phys. 9, 158 (1942).

12. W. J. Popiel Sci. Prog. 58, 237 (1970).

13. H. E. Ries, Jr., Encyclopaedia of Science and Technology, (3rd ed., McGraw-Hill, New York, 1971).

14. T. J. Davies and E. K. Rideal, Interfacial Phenomena (Academic Press, New York, 1961).

15. B. Mann, and H. Kuhn, J. Appl. Phys. 42, 4398 (1971).

16. V. K. Srivastava, and A. R. Verma, Proc. Phys. Soc. 80 , 222 (1962).

17. R. Sirhatta. and G.D. Scott, Appl. Opt., 10, 2192 (1971).

18. M. A. Baker, Thin Solid Films 8, R13 (1971).

19. M. H. Nathoo Thin Solid Films 16, 215 (1973).

20. I. Langmuir Proc. R. Soc. A 170, 1 (1939).

21. S. Tolansky. An Introduction to Interferometry, (Longmans Green, London, 1955) 65.

22. J. Strong, Modern Physical Laboratory Practice (Blackie, London, 1940).

23. H. Bücher, O. V. Elsner, D. Möbius, P. Tillmann and J. Wiegand, Z. Phys. Chem. 65, 152 (1969).

24. I. Langmuir, J. Am. Chem. Soc. 39, 1860 (1917).

25. P. P. Luff and M. Wh ite, Vacuum 18, 437 (1968).

26. V. K. Srivastava, and A. R. Verma, Solid State Commun. 4, 367 (1966).

27. R. W. Roberts and G. L Gains, Jr., Trans. Ninth Natl. Vacuum Symp., Am. Vacuum Soc., 1962, 515.

28. G. L. Gains, Jr. and R. W. Roberts, Nature 197, 787 (1963).

29. L. Holt, Nature 214, 1105 (1967).

30. B. L. Henke, Adv. X-ray Anal. 7, 460 (1964).

31. 1. H. Germer, and K. H. Storks, J. Chem. Phys. 6, 280 (1938).

32. K. Tanaka, Mem. Coll. Sci. Univ. Kyoto A22, 377 (1939).

33. K. G. Brummage, Proc. R. Soc. A 191, 243 (1947).

34. J. M. Cowley, Trans. Farady Soc. 44, 60 (1948).

35. J. W. Menter and D. Tabor, Proc. R. Soc. A. 204, 514 (1950).

36. H. J. Trurnit and G. Schidlovsky, Proc. European Regional Conf. Flectron Microscopy 1960, 2, 721 (1961).

37. G. Knot t, J. H. Schulman and A. l: Wells, Proc. R. Soc. A. 176, 534 (1940).

38. G. Marc and J. Messier, J. Appl. Phys. 45, 2832 (1974).

39. H. T. Ipstein. J. Phys. Colloid Chem. 54, 1053 (1950).

40. H. E. Ries, Jr. and W. A. Kimball, J. Phys. Chem. 59, 94 (1955).

41. H. E Ries, Jr., Sci. Am. 204, 152 (1961).

42. R. M Handy and L. (' Scala, J. Electrochem. Soc. 113, 109 (1966).

43. H. H. Race and S. I. Reynolds, J. Am. Chem. Soc. 61 , 1425 (1939).

44. H Sobotka, M Demeny and J. D. (hauley, J. Colloid Sci. 13, 565 (1958).

45. A. Barraud, J. Messier, A. Rosillo and J. Tanguy, Colloque AVISEM, V'ersailles (Sept. 1971).

46. F.. I. Porter and J. Wyman, Jr., J. Am. Chem. Soc. 60, 1083 (1938).

47. S. I. Reynolds and H.H. Race, G.E. Re'v. 41, 529 (1938).

48. K B. Blodgett, Use of Interference to Extinguish Reflection of Light from (ilass (unpublished).

49. C. E. Buckwald, D. M. Gallagher, C.P. Haskins, E. M.
Thatcher and P. A. Zahl, Proc. Natl. Acad. Sci. 24, 204 (1938).

50. P A. Zahl, C. P. Haskins, D. M. Gallagher and C. E. Buckwald, Trans. Faraday Soc. 35, 308 (1939).

51. R. W Goranson and W. A. Zisman, J. Chem. Phys. 7, 492 (1939).

52. K. H. Beck, Ph. D. Thesis, University of Marburg, W. Germany, 1966 (unpublished)

53. K. H. Drexhage and H. Kuhn, Basic Problems in Thin Film Physics, (Göttingen, Vandenhoeck and Ruprecht, 1966) 339.

54. P. L. Hawkes, Thin Film Integrated Circuits, (AEI Engineering) 5, 304 (1965).

55. A Leger. J. Klein, M. Belin and D. Defourneau, Thin Solid Films 8, R51 (1971).

56. U. Khanna and V. K. Srivastava, Thin Solid Films 12, S25 (1972).

57. U. Khanna, V. K. Srivastava and V. K. Agarwal, Thin Films 2, 83 (1971).

58. U. Khanna, and V. K. Srivastava, Thin Films 2, 167 (1972).

59. U. Khanna, Ph. D. Thesis, University of Roorkee, India, 1973 (unpublished).

60. K. L. Chopra, J. Appl. Phys. 36, 655 (1963).

61. H. E. Ries, Jr. and D. C, Walker, J. Colloid Sci. 16, 361 (1961).

62. H. E. Ries, Jr. and W. A. Kimball, Nature 181. 901 (1958).

63. J. Tanguy and P. Hesto, Thin Solid Films 21, 129 (1974)

64. H. Müller, Phys. Rev. 50, 547 (1936).

65. L. Salem, J. Chem. Phys. 37, 2100 (1962).

66. K. G. Denbigh, Trans. Faraday Soc. 36, 936 (1940).

67. C. P. Smyth, Dielectric Constant and Molecular Structure, (Chem. Catalog., New York, 1931).

68. H. Fröhlich, Theory of Dielectrics (Oxford Univ. Press, 1958) 2nd Ed.

69. J. J. O'Dwyer, The Theory of Electrical Conduction and Breakdown in Solid Dielectrics, (Clarendon Press, Oxford, 1973).

70. R. H. Fowler and I. Nordheim, Proc. R. Soc. A 119, 173 (1928).

71. J. Frenkel, Phys. Rev. 36, 1604 (1930).

72. A. Sommerfeld and H. Bethe, Handbuch der Physik, $X X I V$, Pt. 2, (Julius Springer, Berlin, 1933) 133.

73. J. G. Simmons, D.C. Conduction in Thin Films, (Mills and Boon, L.ondon, 1971).

74. W. Schottky Phys. Z. 15, 872 (1914).

75. N. Mott and R. W. Gurney, Electronic Processes in Solids (Oxford University Press, London, 1948).

76. A Rose, Phys. Rev. 97, 1538 (1955).

77. J. Hrenkel, Tech. Phys. USSR 5, 685 (1938); Phys. Rev. 54, 647 (1938)

78. C. A. Mead, Phys. Rev. 128, 2088 (1962).

79. J. Giaver, Phy's. Rev. Lett. 5, 147 (1960); 5, 464 (1960).

80. J. Nicol, S. Shapiro and P. Smith, Phys. Rev. Lett. 5, 461 (1960)

81. J. L. Miles, and H. O. McMahon, J. Appl. Phys. 32, 1176 (1961).

82. R. Stratton, J. Phy's. \& Chem. Sol. 23, 1177 (1962)

83. J. G. Simmons, J. Appl. Phys. 34, 1793 (1963); 34, 2581 (1963).

84. S. Horiuchi. J. Yamaguchi and K. Naito, J. Electrochem. Soc. 115, 634 (1968).

85. J. G. Simmons, J. Appl. Phys. 35, 2472 (1964).

86. S. R. Pollack and ('.E. Morris, J. Appl. Phys. 35, 1503 (1964). 
87. W. E. Flannery and S R. Pollack, J. Appl. Phys. 37, 4417 (1966).

88. B. Mann. H. Kuhn and L.v. Szentpaly, Chem. Phys. Lett. $8,82(1971)$

89. L. v. Szentpaly, D. Möbius and H. Kuhn, J. Chem. Phys. 52, 4618 (1970).

90. V. K. Srivastava, Phys. Rev. Lett. 30, 1046 (1973).

91. M. H. Nathoo and A. K. Jonscher, J. Phys. C 4, L301 (1971).
92. R. M. Hill, Phil. Mag. 23, 59 (1971).

93. A. K. Jonscher, J. Vac. Sci \& Technol. 8, 135 (1971).

94. A. K. Jonscher, Phil. Mag. 23, 205 (1971).

95. A. K. Jonscher, J. Noncryst. Solids 8- 10, 293 (1972).

96. A. K. Jonscher and M. H. Nathoo, Thin Solid Films 12, S15 (1972).

97. A K. Jonscher, J. Electrochem. Soc. (To be published).

98. E. J. Le Sueur and A. K. Jonscher, Thin Solid Films 12, 59 (1972). 

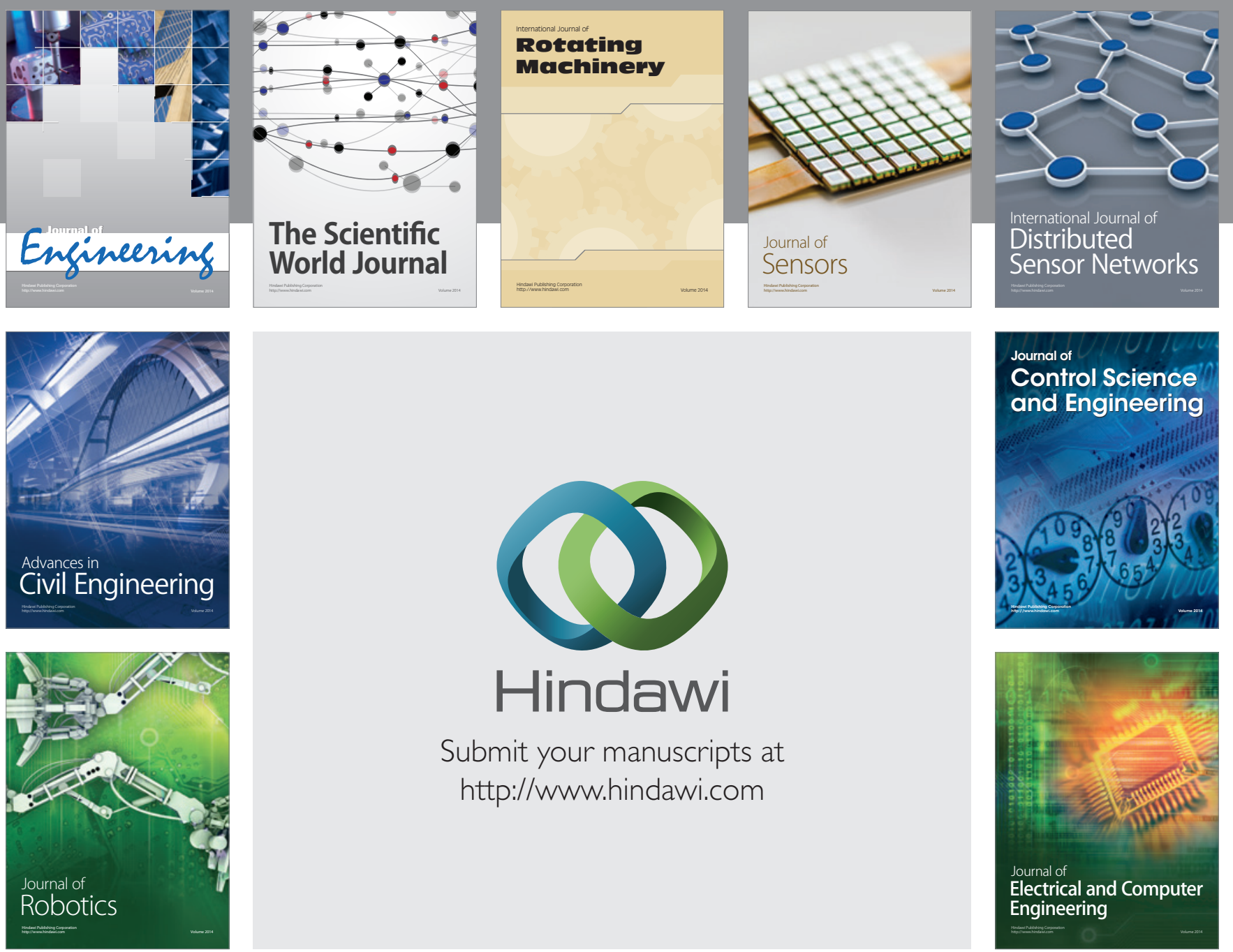

Submit your manuscripts at

http://www.hindawi.com
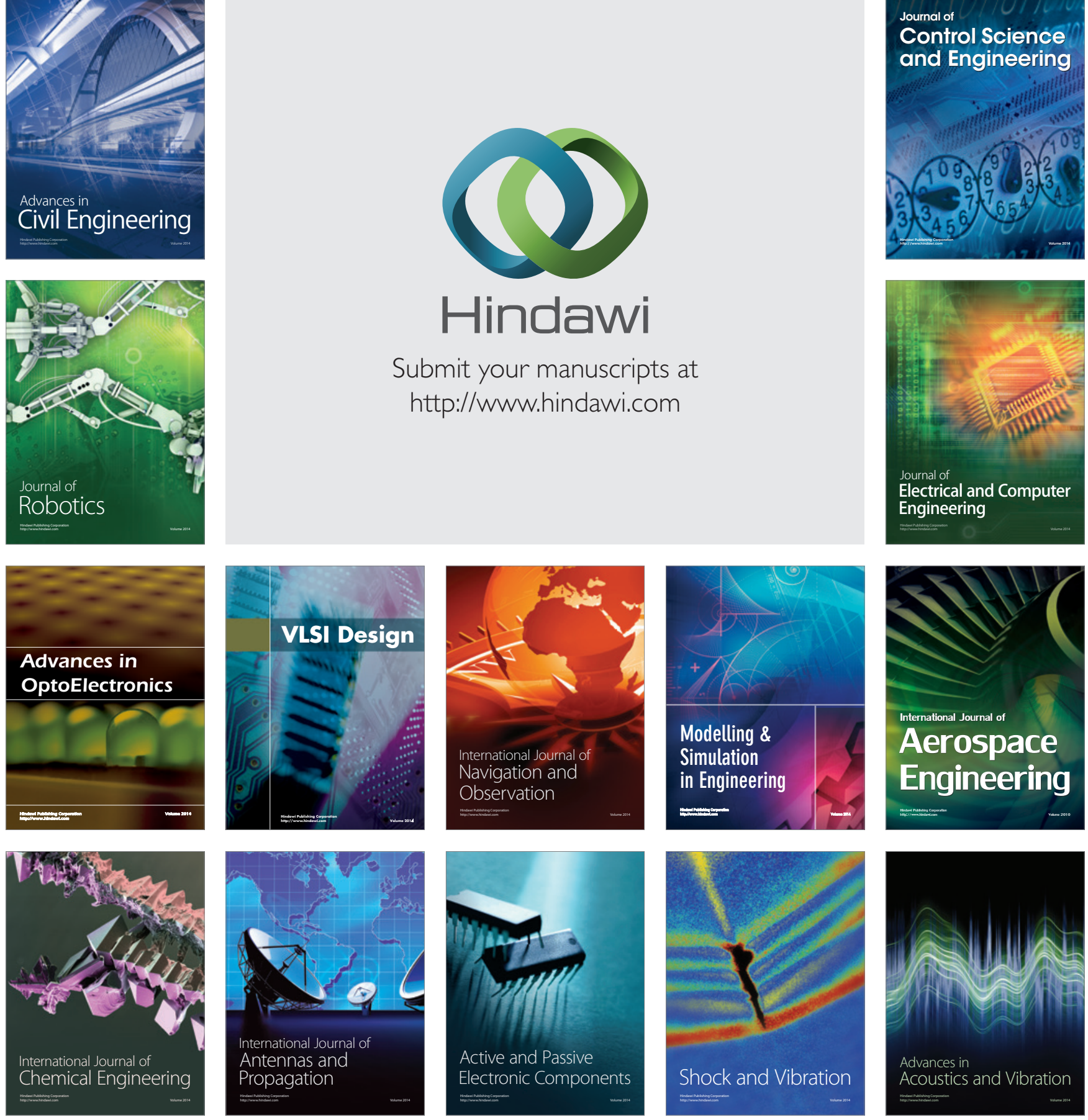